\title{
A New Gene Set Identifies Senescent Cells and Predicts Senescence-Associated Pathways Across Tissues
}

Abbreviated Title: A new gene set identifies senescent cells

Authors: Dominik Saul, M.D. ${ }^{1,2,3}$, Robyn Laura Kosinsky, Ph.D. ${ }^{4}$, Elizabeth J Atkinson, M.S. ${ }^{5}$, Madison L. Doolittle, Ph.D. ${ }^{1,2}$, Xu Zhang, Ph.D., ${ }^{2,6}$ Nathan K. LeBrasseur, Ph.D. 2,6, Robert J. Pignolo, M.D., Ph.D ${ }^{1,2,6}$, Paul D. Robbins, Ph.D.7, Laura J. Niedernhofer, M.D., Ph.D.7, Yuji Ikeno, M.D., Ph.D. ${ }^{8}$, Diana Jurk, Ph.D., ${ }^{2,6}$ João F. Passos, Ph.D. ${ }^{2,6}$, LaTonya J. Hickson, M.D. ${ }^{9}$, Ailing Xue, M.D. ${ }^{2}$, David G. Monroe, Ph.D. ${ }^{1,2}$, Tamara Tchkonia, Ph.D., M.S. ${ }^{2,6}$, James L. Kirkland, M.D., Ph.D. ${ }^{2,6}$, Joshua N. Farr, Ph.D. ${ }^{1,2,6^{*}}$ and Sundeep Khosla, M.D. ${ }^{1,2,6^{*}}$

\author{
Affiliations: \\ ${ }^{1}$ Division of Endocrinology, Mayo Clinic, Rochester, MN, 55905, USA. \\ ${ }^{2}$ Robert and Arlene Kogod Center on Aging, Mayo Clinic, Rochester, MN 55905, USA. \\ ${ }^{3}$ Department of Trauma, Orthopedics and Reconstructive Surgery, Georg-August-University of \\ Goettingen, Germany. \\ ${ }^{4}$ Division of Gastroenterology and Hepatology, Mayo Clinic, Rochester, MN, 55905, USA. \\ ${ }^{5}$ Department of Quantitative Health Sciences, Mayo Clinic, Rochester, MN, USA. \\ ${ }^{6}$ Department of Physiology and Biomedical Engineering, Mayo Clinic, Rochester, MN, USA. \\ ${ }^{7}$ Institute on the Biology of Aging and Metabolism, Department of Biochemistry, Molecular Biology \\ and Biophysics, University of Minnesota, Minneapolis, MN, USA. \\ ${ }^{8}$ Department of Pathology, University of Texas Health, San Antonio, TX, USA. \\ ${ }^{9}$ Division of Nephrology and Hypertension, Mayo Clinic, Jacksonville, FL, USA.
}

Funding: This work was supported by the German Research Foundation (D.F.G., 413501650) (D.S.), National Institutes of Health (NIH) grants P01 AG062413 (S.K., J.N.F., N.K.L., R.P., P.D.R., L.J.N., Y.I., J.P., D.G.M., T.T., J.L.K.), R21 AG065868 (S.K., J.N.F), K01 AR070241 (J.N.F.), R01 AG063707 (D.G.M.), R37 AG 013925 (J.L.K., T.T.), R33AG 61456 (J.L.K., T.T., R.P., P.D.R., L.J.N., S.K.), 1R01AG068048-01 (JFP), R56 AG 60907 and R01 AG55529 (N.K.L.)., the Connor Fund (J.L.K., T.T.), Robert P. and Arlene R. Kogod (J.L.K.), Robert J. and Theresa W. Ryan (J.L.K., T.T.), the Noaber Foundation (J.L.K., T.T.), and Mildred Scheel postdoc fellowship by the German Cancer Aid (R.L.K.).

\section{Emails:}

- Dominik Saul: saul.dominik@mayo.edu; ORCiD: 0000-0002-0673-3710

- Robyn Laura Kosinsky: kosinsky.robynlaura@mayo.edu; ORCiD: 0000-0003-2869-7762

- Elizabeth J Atkinson: Atkinson@mayo.edu; 0000-0002-1191-3775

- Madison L. Doolittle: doolittle.madison@mayo.edu; ORCiD: 0000-0003-0912-0095

- Xu Zhang; zhang.xu@mayo.edu; ORCiD: 0000-0002-9784-0481

- Nathan LeBrasseur: LeBrasseur.Nathan@mayo.edu; ORCiD: 0000-0002-2002-0418

- Robert J. Pignolo: Pignolo.Robert@mayo.edu

- Paul Robbins: probbins@umn.edu; ORCiD: 0000-0003-1068-7099

- Laura Niedernhofer, Iniedern@umn.edu; ORCiD: 0000-0002-1074-1385

- Yuji lkeno: Ikeno@uthscsa.edu

- Diana Jurk: jurk.diana@mayo.edu

- João Passos: Passos.Joao@mayo.edu; ORCiD: 0000-0001-8765-1890

- LaTonya J. Hickson: Hickson.Latonya@mayo.edu

- Ailing Xue: Xue.Ailing@mayo.edu

- David G. Monroe: Monroe.David@mayo.edu; ORCiD: 0000-0002-4818-0114 
- Tamara Tchkonia: Tchkonia.Tamar@mayo.edu; ORCiD: 0000-0003-4623-7145

- James Kirkland: Kirkland.James@mayo.edu; ORCiD: 0000-0003-1676-4905

- Joshua N. Farr: farr.joshua@mayo.edu; ORCiD: 0000-0002-3179-6414

- Sundeep Khosla: khosla.sundeep@mayo.edu; ORCiD: 0000-0002-2936-4372

\section{${ }^{*}$ Corresponding authors:}

*Joshua N. Farr, Ph.D., Guggenheim 7-11D, Mayo Clinic College of Medicine, 200 First Street SW, Rochester, MN 55905; Tel. +1-507-538-0085; Fax. +1-507-284-9111; Email: farr.joshua@mayo.edu

*Sundeep Khosla, M.D., Guggenheim 7-11, Mayo Clinic College of Medicine, 200 First Street SW, Rochester, MN 55905; Tel: +1-507-255-6663; Email: khosla.sundeep@mayo.edu

Key Words: bone, senescence, SASP, gene set, aging

Supplementary Material: This manuscript contains Supplementary Methods and Figures

Disclosures: Patents on senolytic drugs and their uses and on SASP biomarkers are held by Mayo Clinic and the University of Minnesota. This research has been reviewed by the Mayo Clinic Conflict of Interest Review Board and was conducted in compliance with Mayo Clinic Conflict of Interest policies. 


\section{Abstract}

Although cellular senescence is increasingly recognized as driving multiple age-related co-morbidities through the senescence-associated secretory phenotype (SASP), in vivo senescent cell identification, particularly in bulk or single cell RNA-sequencing (scRNA-seq) data remains challenging. Here, we generated a novel gene set (SenMayo) and first validated its enrichment in bone biopsies from two aged human cohorts. SenMayo also identified senescent cells in aged murine brain tissue, demonstrating applicability across tissues and species. For direct validation, we demonstrated significant reductions in SenMayo in bone following genetic clearance of senescent cells in mice, with similar findings in adipose tissue from humans in a pilot study of pharmacological senescent cell clearance. In direct comparisons, SenMayo outperformed all six existing senescence/SASP gene sets in identifying senescent cells across tissues and in demonstrating responses to senescent cell clearance. We next used SenMayo to identify senescent hematopoietic or mesenchymal cells at the single cell level from publicly available human and murine bone marrow/bone scRNA-seq data and identified monocytic and osteolineage cells, respectively, as showing the highest levels of senescence/SASP genes. Using pseudotime and cellular communication patterns, we found senescent hematopoietic and mesenchymal cells communicated with other cells through common pathways, including the Macrophage Migration Inhibitory Factor (MIF) pathway, which has been implicated not only in inflammation but also in immune evasion, an important property of senescent cells. Thus, SenMayo identifies senescent cells across tissues and species with high fidelity. Moreover, using this senescence panel, we were able to characterize senescent cells at the single cell level and identify key intercellular signaling pathways associated with these cells, which may be particularly useful for evolving efforts to map senescent cells (e.g., SenNet). In addition, SenMayo represents a potentially clinically applicable panel for monitoring senescent cell burden with aging and other conditions as well as in studies of senolytic drugs. 


\section{Introduction}

Cellular senescence is now recognized as a fundamental mechanism of aging in animals and humans. Accumulation of DNA damage and/or other cellular stressors ${ }^{1-4}$ causes proliferating ${ }^{5,6}$ as well as terminally differentiated, non-dividing cells ${ }^{7-10}$ to undergo senescence. Characteristics of senescent cells include profound chromatin and secretome changes, along with increased expression of a number of senescence markers, including Cdkn2a/p16 Ink4a and Cdkn1a/p2 $1^{\text {Cip } 1}$, immune evasion, and resistance to apoptosis. ${ }^{1,11}$ Senescent cells can develop a senescence-associated secretory phenotype (SASP), consisting of pro-inflammatory cytokines, chemokines, extracellular matrix-degrading proteins, and other factors that have deleterious paracrine and systemic effects. ${ }^{12-15}$ Further, because senescent cells accumulate in multiple tissues in temporal and spatial synchrony with age-associated functional decline in both animals and humans,,$^{5,6,16}$ they have been hypothesized to drive the deterioration linked to numerous chronic diseases. ${ }^{1}$

Importantly, the SASP as a feature of cellular senescence represents not just a locally or systemically detrimental set of factors that, in the aging organism, cause physical, metabolic, and cognitive decline, ${ }^{17-21}$ but is also a therapeutic target of interest. ${ }^{22-24}$ Thus, given the broad availability of next-generation sequencing, there is considerable interest in monitoring responses to senolytic treatments. However, this has been challenging, especially at the single cell level. ${ }^{25}$ In part, this is due to an imprecise definition of the heterogeneous population of senescent cells and their associated SASP which complicates appropriate monitoring of senescent cell clearance.

Due to variations in the composition of a "senescence gene set" in the current literature, in the present study we sought to identify commonly regulated genes in various age-related data sets in a transcriptome-wide approach that included whole-transcriptome as well as single cell RNA-sequencing (scRNA-seq) ${ }^{26}$ Based on an extensive review of the literature, we defined a panel of 125 genes as our senescence gene set ("SenMayo"), which we then validated in our own as well as publicly available datasets of tissues from aged humans and mice, including changes 
in this gene set following the clearance of senescent cells. Recognizing the difficulty of identifying senescent cells within scRNA-seq analyses, we next applied SenMayo to available scRNA-seq data from human and murine bone marrow/bone hematopoietic and mesenchymal cells, ascertained the identity of the senescent cells in these analyses, and characterized the communication patterns of senescent hematopoietic or mesenchymal cells with other cells in their microenvironment. Finally, we experimentally validated key predictions from our in silico analyses in a mouse model of aging and following genetic clearance of senescent cells. 


\section{Results}

Development and validation of SenMayo in human datasets. We first analyzed previously published $^{27,28}$ as well as unpublished (see Methods) transcriptome-wide mRNA-seq analyses of human whole bone biopsies. These included bone and bone marrow (Cohort A) ${ }^{27}$ as well as bone biopsies that were processed to remove bone marrow and bone surface cells and were thus highly enriched for osteocytes (Cohort $B)^{28}$ from young vs. elderly women (Fig. 1A). We used transcriptional regulatory relationships ${ }^{29}$ to evaluate whether senescence- and SASP-associated pathways were enriched with aging in humans and noted enrichment of genes regulating inflammatory mediators, including NFKB1, RELA, and STAT3 (Fig. 1B). As expected, both aged cohorts displayed an upregulation of senescence- and SASP markers such as CDKN1A/p21 ${ }^{\text {cip1, }}$, CCL2, and IL6 (Fig. 1C). It should be noted that some canonical markers of senescence, including $C D K N 2 A / p 16^{\text {Ink4a }}$, did not show the predicted increase with aging due to comparatively low expression levels. Given the limitations of single gene analyses to predict the complex mechanisms of cellular aging, we next tested whether a previously published combination of senescence/SASP genes (R-HSA-2559582) is enriched in our aging cohorts. However, this Gene Set Enrichment (GSEA)-based approach failed to predict an age-related senescence/SASP increase in either cohort (Fig. 1D). 
A
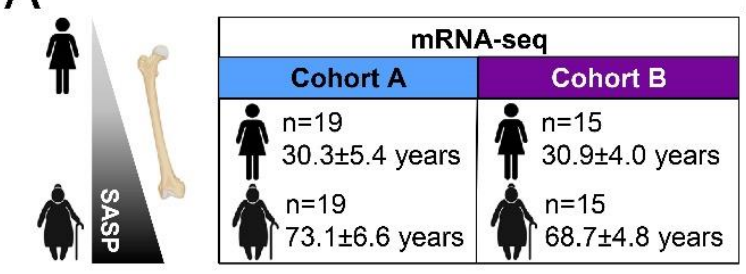

C
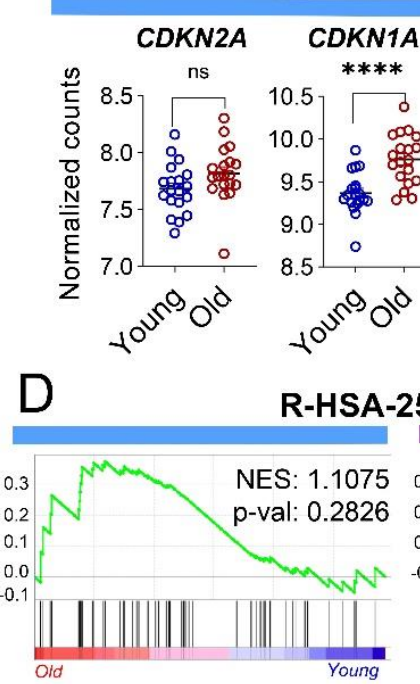

$\mathrm{E}$

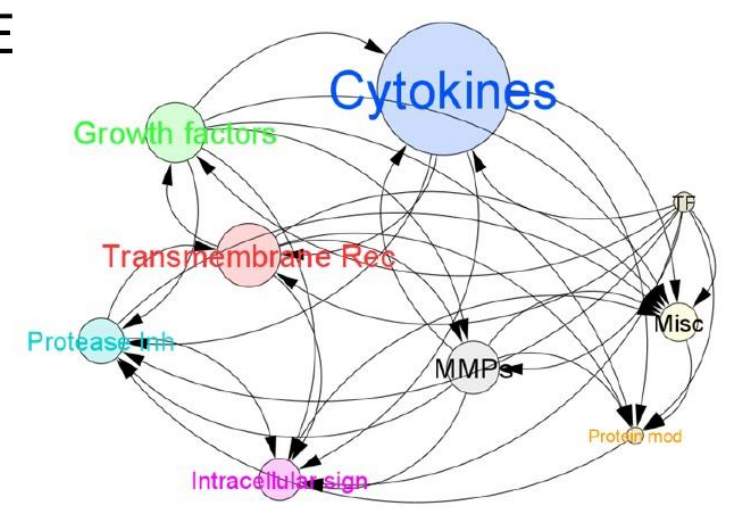

B

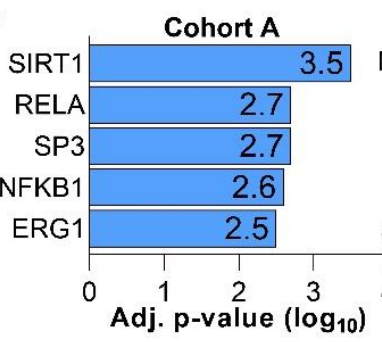

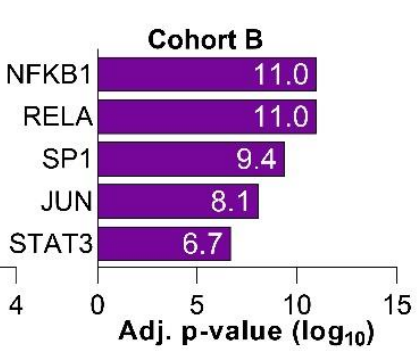
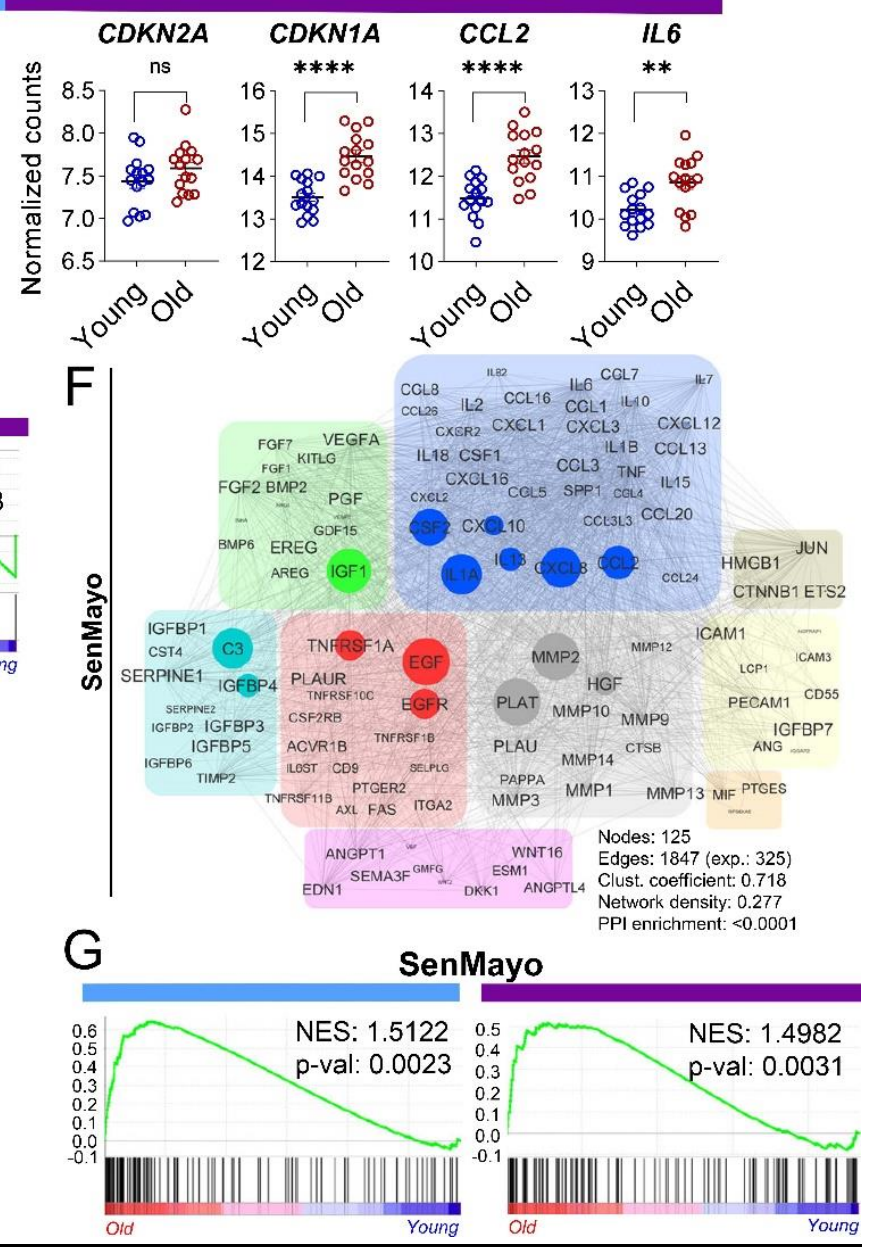

Figure 1. Development and validation of the SenMayo gene set. (A) Human samples from Cohort $\mathrm{A}$ (bone and bone marrow biopsies) and cohort $\mathrm{B}$ (highly enriched osteocyte fractions) were used for transcriptome-wide RNA-seq analyses; (B) Making use of TRRUST analyses, ${ }^{30}$ we found several inflammation- and stress-associated genes, including SIRT1 and NFKB1, to be upregulated in the elderly women; (C) In both gene sets, CDKN1A/P21 ${ }^{\text {Cipt }}$ and several SASP markers such as CCL2 and IL6 showed consistent upregulation with aging, while $C D K N 2 A / p 16^{\text {Ink4a }}$ (due to comparatively low expression) did not change significantly; (D) The commonly used senescence/SASP gene set (R-HSA-2559582) failed to predict the aging process in either human cohort; $(E)$ The SenMayo gene set includes growth factors, transmembrane receptors, and cytokines/chemokines that are highly influenced by other members of the gene set. The circle size depicts groupwise interactions; (F) SenMayo encodes a dense network of nine different protein classes within a strong interaction network. The size of each circle represents 
the connectivity with other members of the gene set; ${ }^{30}(\mathrm{G})$ Genes included in the SenMayo gene set were significantly enriched with aging in both human cohorts. Cohort $A: n=38$ (19 young, 19 old, all + ), Cohort $B: n=30$ (15 young, 15 old, all ${ }_{+}$). ${ }^{* *} p<0.01,{ }^{* * *} p<0.0001$. Figure $1 A$ was designed using Biorender.com.

In order to develop a more robust gene panel associated with cellular senescence, we next generated a novel gene set to predict the expression of aging-related senescence genes by performing an in-depth, rigorous literature search (see Methods for details of how these genes were selected). The result was a novel senescence gene set of 125 genes (SenMayo) that consisted predominantly of SASP factors $(n=83)$ but also included transmembrane $(n=20)$ and intracellular $(n=22)$ proteins (Table 1). Within this SenMayo gene set, which comprised 9 distinct clusters, cytokines/chemokines were the most densely connected regulators according to the number of descendent proteins in STRING analysis (Fig. 1E, F). Predominant connectivity (whole network density: 0.277, PPI <0.0001) was shown by IL1A, CXCL8, CCL2 (cytokines/chemokines, blue), IGF1 (growth factor, green), C3 and IGFBP4 (protease inhibitor, turquoise), TNFRSF1A, EGF and EGFR (transmembrane signal receptors, red), and MMP2, PLAT, and HGF ([metallo]proteases, grey) (Fig. 1F). Notably, when testing the enrichment of SenMayo within our two human mRNA-seq cohorts, senescence/SASP genes were significantly enriched in the bone samples obtained from elderly women ( $p=0.002$ [Cohort $A$ ] and $p=0.003$ [Cohort B]; Fig. 1G). Using Cohort A as an example, within the R-HSA-2559582 gene set, 2 out of 50 available genes were significantly enriched in the biopsies from elderly women (Suppl. Fig. 1A), while 13 out of 120 available genes of the SenMayo gene set were significantly enriched in the elderly women (Suppl. Fig. 1B). Note that the GSEA analysis includes not only genes that differ significantly between groups, but also evaluates overall trends for differences in gene expression between groups and hence provides considerably greater power than examining individual genes. ${ }^{31}$ The canonical SASP markers CCL24, SEMA3F, FGF2, and IGFBP7 were consistently enriched in Cohort A (Suppl. Fig. 1C) and Cohort B (Suppl. Fig. 1D). In addition, SEMA3F was significantly correlated with the senescence marker, $C D K N 1 A / p 21^{\text {Cip } 1}$, in both cohorts (Suppl. Fig. 1E, F). 
SenMayo is applicable across tissues and species. To evaluate the applicability of SenMayo across tissues and species, we next analyzed publicly available mRNA-seq data from brain tissue isolated from young vs. aged mice (GSE145265 32 , GSE128770 33 , GSE94832 34 , Fig. 2A-C). As is evident, aged mouse brain cells (microglia) and regions (prefrontal cortex, dorsal hippocampus) displayed a highly significant enrichment of senescence/SASP genes using the SenMayo gene list $(p=0.005, p=0.001, p<0.001$, respectively), while the previously published gene set (R-HSA2559582) did not reach statistical significance $(p=0.157, p=0.117, p=0.192$, respectively). In addition, using murine bone marrow from the tabula muris senis (a murine single cell transcriptome atlas of young vs. aged tissues ${ }^{35}$ ), the applicability of SenMayo in predicting the aging process was confirmed by GSEA (Fig. 2D) Thus, SenMayo identifies senescent cells associated with aging across tissues (bone/bone marrow and brain) and species (humans and mice).
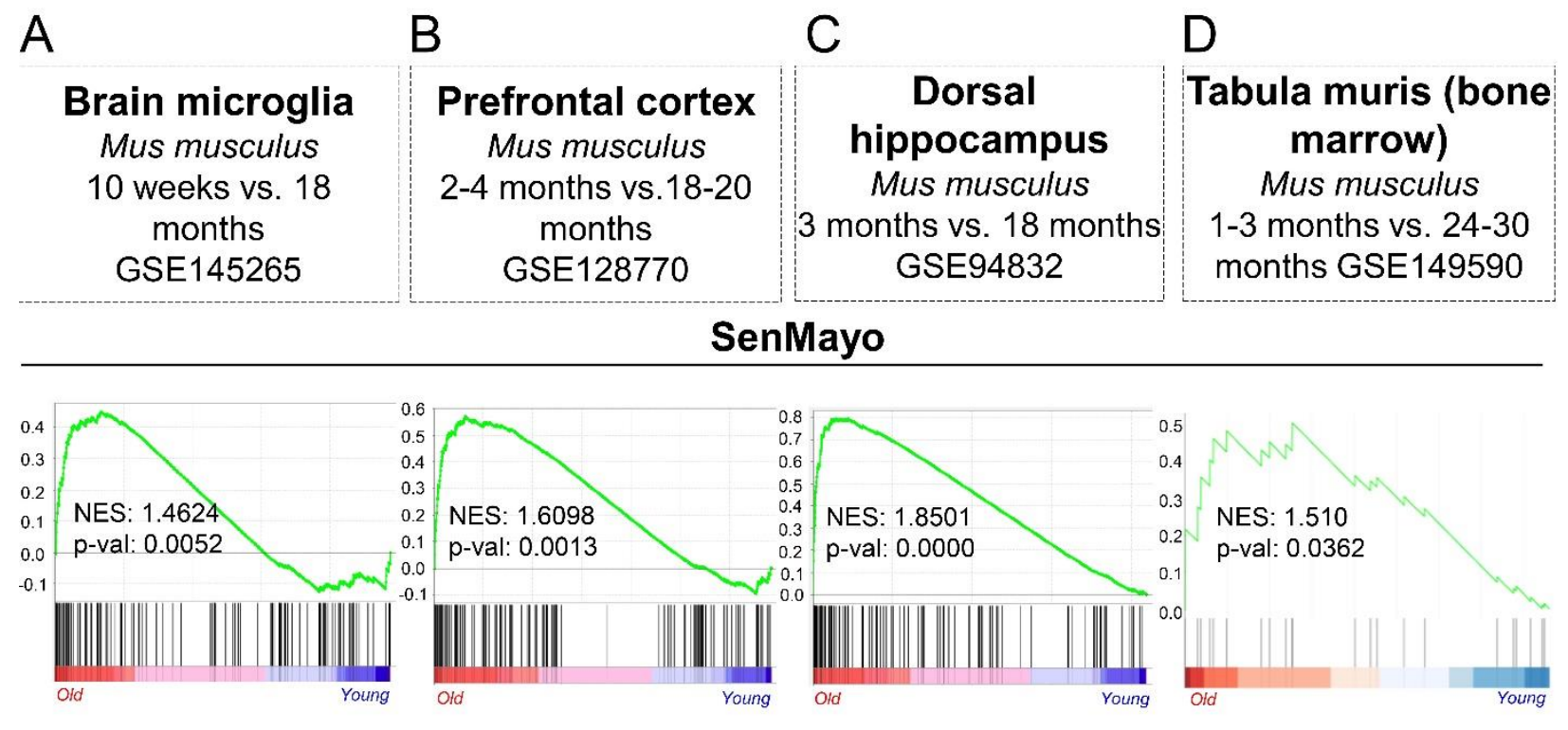

\section{R-HSA-2559582}

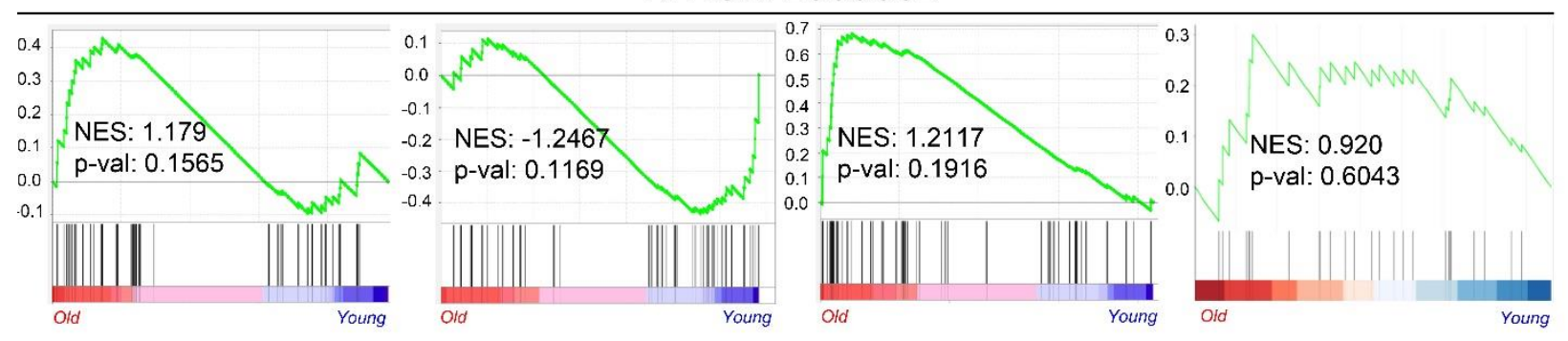


Figure 2. The SenMayo gene set predicts aging across tissues and species. (A) Compared to the conventional gene set, the SenMayo list is significantly enriched during the aging process in murine brain microglia ( $p=0.1565$ vs. $p=0.0052$; GSE145265), $n=4$ (2 young, 2 aged, all $\precsim$ ), (B) murine prefrontal cortex ( $\mathrm{p}=0.1169$ vs. $\mathrm{p}=0.0013$; GSE128770), $\mathrm{n}=48$ (24 young, 24 aged, all $\widehat{)}$ ), and $(\mathrm{C})$ murine dorsal hippocampus ( $\mathrm{p}=0.1916$ vs. $\mathrm{p}<0.001$; GSE94832), $\mathrm{n}=12$ (6 young (3 + ), 6 aged (2). Likewise, the murine bone marrow (D) within the tabula muris senis (GSE149590 ${ }^{36}$ ) has a higher enrichment of the SenMayo genes within the old cohort ( $p=0.6043$ vs. $p=0.0362$ ),

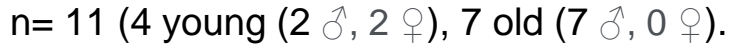

SenMayo not only predicts aging, but also demonstrates clearance of senescent cells. In order to independently validate our in silico analyses, we next made use of our previously described $p 16$ INK-ATTAC mouse model that allows for inducible clearance of $p 16^{\text {Ink4a }}$-expressing senescent cells after administration of the drug AP20187 (AP). ${ }^{37}$ In previous studies, we have demonstrated increases in Cdkn2a/p16 ${ }^{\text {Ink4a }}$ and Cdkn1a/p21 ${ }^{\text {Cip1 }}$ mRNA levels with aging in bones from these mice $^{7}$ as well as reductions in these mRNAs following clearance of senescent cells in p16-INKATTAC mice treated with AP and concordant changes in other markers of cellular senescence (e.g., telomeric DNA damage markers in osteocytes). ${ }^{37}$ Importantly, in young vs. old mice, SenMayo was expressed at a significantly higher level in bones from the old mice (Fig. 3A) and was significantly reduced following AP treatment of old p16-INK-ATTAC mice (Fig. 3B). Moreover, by using the SenMayo genes, a higher overlap of young vs. old + AP-treated mice as compared to young vs. old + vehicle-treated mice was observed through principal component analysis (PCA) (Fig. 3C). 
A

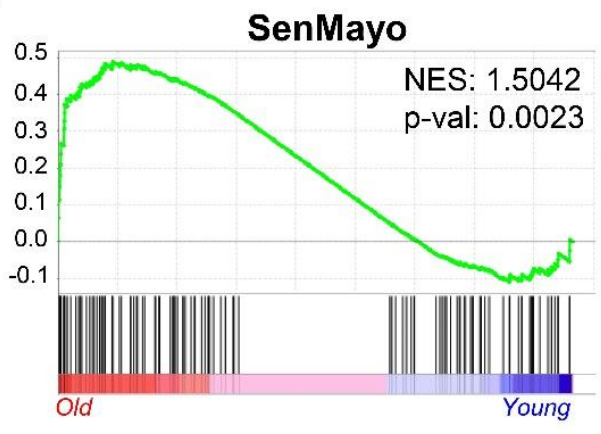

C

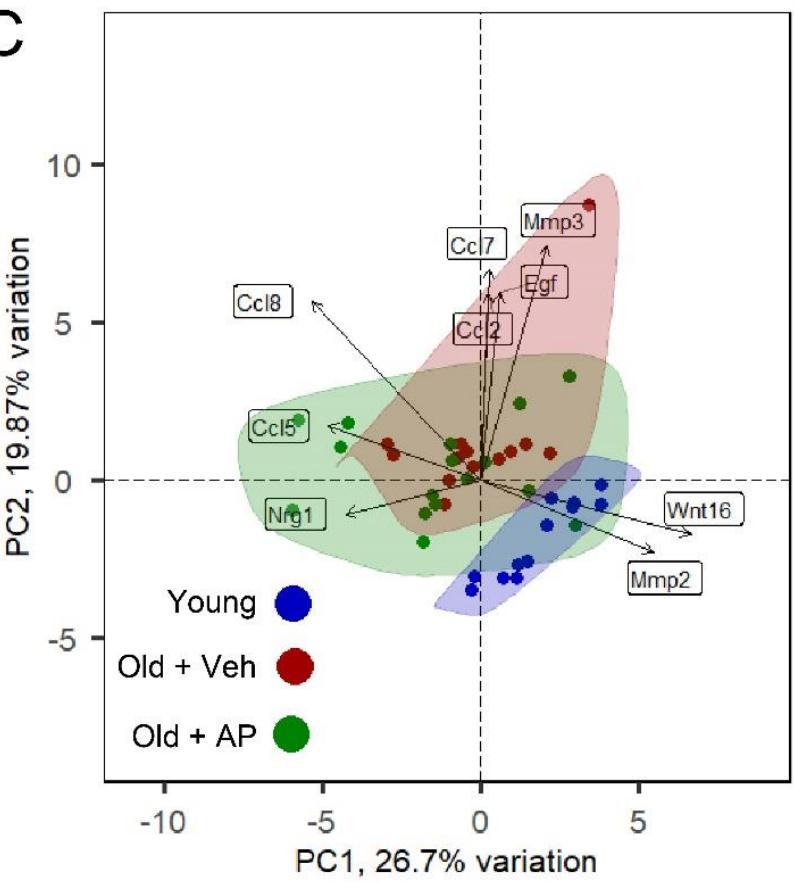

$E$

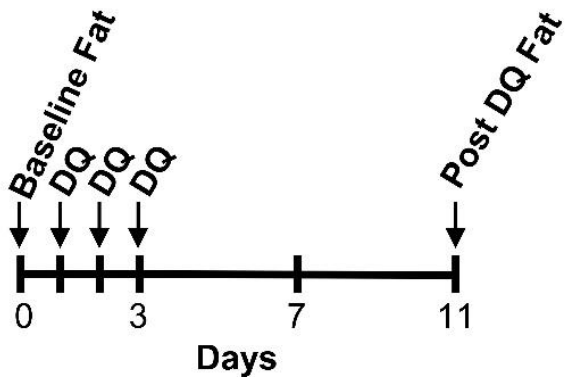

B

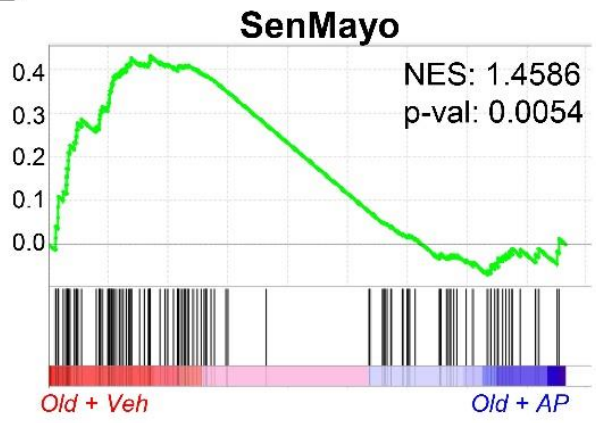

$\mathrm{D}$
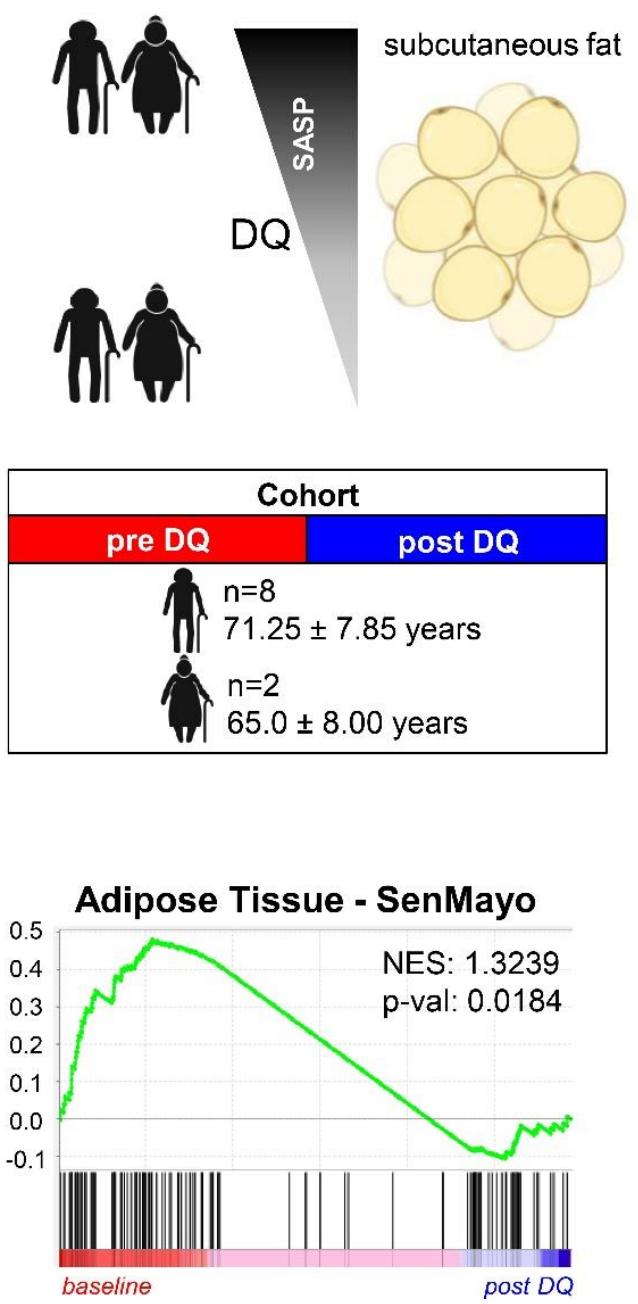

Figure 3. The SenMayo gene set tracks genetic and pharmacologic clearance of senescent cells. (A) The SenMayo panel successfully indicated aging in bone in mice ( $p$-value $=0.0023), n=25$ (12 young, 13 old (all ) ); (B) The elimination of $p 16^{\text {Ink4a }}$-expressing senescent cells by AP20187 administration was shown previously to reverse the aging bone phenotype. ${ }^{37}$ The SenMayo gene set successfully demonstrated the significant reversal of the aging phenotype at the gene expression level upon the elimination of $p 16^{\text {lnk4a }}$-expressing senescent cells $(p=0.0054), n=29$ (13 Veh, 16 AP (all +); (C) By specifically using the expression patterns of the SenMayo gene set, 
our bone RNA-seq revealed no similarities in gene expression patterns between young and old + veh treated mice, and a substantial overlap of expression profiles of old + AP mice with young mice; (D) We used a previously published mRNA-seq dataset from human adipose tissue of our group, ${ }^{24,38}$ to evaluate changes in SenMayo following $D+Q$ treatment. Adipose tissue was collected before and 11 days after three days of oral $D+Q$ treatment. Figure was designed using Biorender.com; (E) Using SenMayo, there was a was a reduction of SenMayo $(p=0.0184)$ in the subcutaneous fat samples in the subjects treated with $D+Q$, consistent with a reduction in senescent cell burden following $D+Q$ treatment $(n=10(8 \stackrel{0}{\AA}, 2$ + $))$.

We further validated the ability of SenMayo to predict senescent cell clearance by examining a human cohort. In a phase I pilot study, the senolytic combination of Dasatinib plus Quercetin $(D+Q)^{39}$ was administered to subjects with diabetic kidney disease for 3 consecutive days. ${ }^{24,38}$ We performed RNA-seq from adipose tissue samples obtained from these subjects before and 11 days after $D+Q$ treatment (male: female=9:3, age: 68.8[ \pm 9.3$]$ years:65.3[ \pm 6.6$]$ years, Fig. 3D). ${ }^{24,38}$ As shown in Fig. 3E, there was a significant reduction in SenMayo $(p=0.0184)$ in the subcutaneous adipose tissue samples in the subjects following $D+Q$ treatment, consistent with a reduction in senescent cell burden, which was independently validated by demonstrating reductions in $\mathrm{p} 16^{\mathrm{lnk} 4 \mathrm{a}}+, \mathrm{p} 21^{\mathrm{Cip} 1}+$, and $\mathrm{SA}-\beta \mathrm{gal}+$ cells in the adipose tissue biopsy samples following $D+Q$ treatment. ${ }^{24,38}$ Thus, these direct interventional studies in mice and humans demonstrate that not only is SenMayo associated with aging, but it is also reduced following clearance of senescent cells.

SenMayo outperforms existing senescence/SASP gene sets. In addition to directly comparing SenMayo to the R-HSA-2559582 senescence/SASP gene set, we also compared it to five additional senescence/SASP gene sets ${ }^{40-44}$ in all of the mouse and human models described above. As shown in Table 2, SenMayo consistently outperformed these gene sets (based on normalized enrichment scores [NES] and p-values) both in the ability to identify senescent cells with aging across tissues and species and in demonstrating responses to senescent cell clearance.

The SenMayo gene set identifies senescent hematopoietic and mesenchymal cells within scRNAseq bone marrow/bone datasets. Although scRNA-Seq provides extremely important information 
regarding changes in gene expression at the individual cell level, it has been problematic for evaluating cellular senescence in a given cell. In part, this is because the $C d k n 2 a / p 16^{\text {Ink4a }}$ mRNA is expressed at relatively low levels, even in senescent cells, ${ }^{45}$ and may not be reliably detected in scRNA-seq data. Although Cdkn1a/p21 ${ }^{C i p 1}$ is generally expressed at higher levels in RNA-seq data, presence or absence of $C d k n 1 a / p 21^{\text {Cip } 1}$ also may not consistently identify a senescent cell. ${ }^{42}$ As such, having validated SenMayo as being associated not only with aging but also specifically with cellular senescence, we next tested whether it could identify senescent cells at the single cell level. To evaluate this first for hematopoietic cells, we analyzed publicly available single cell bone marrow datasets from 20 healthy donors across a broad age range (24-84 years) ${ }^{46}$ and evaluated 68,478 hematopoietic cells for expression of the SenMayo gene set (GSE120446), ${ }^{46} \mathrm{Fig} .4 \mathrm{~A}$ ). 


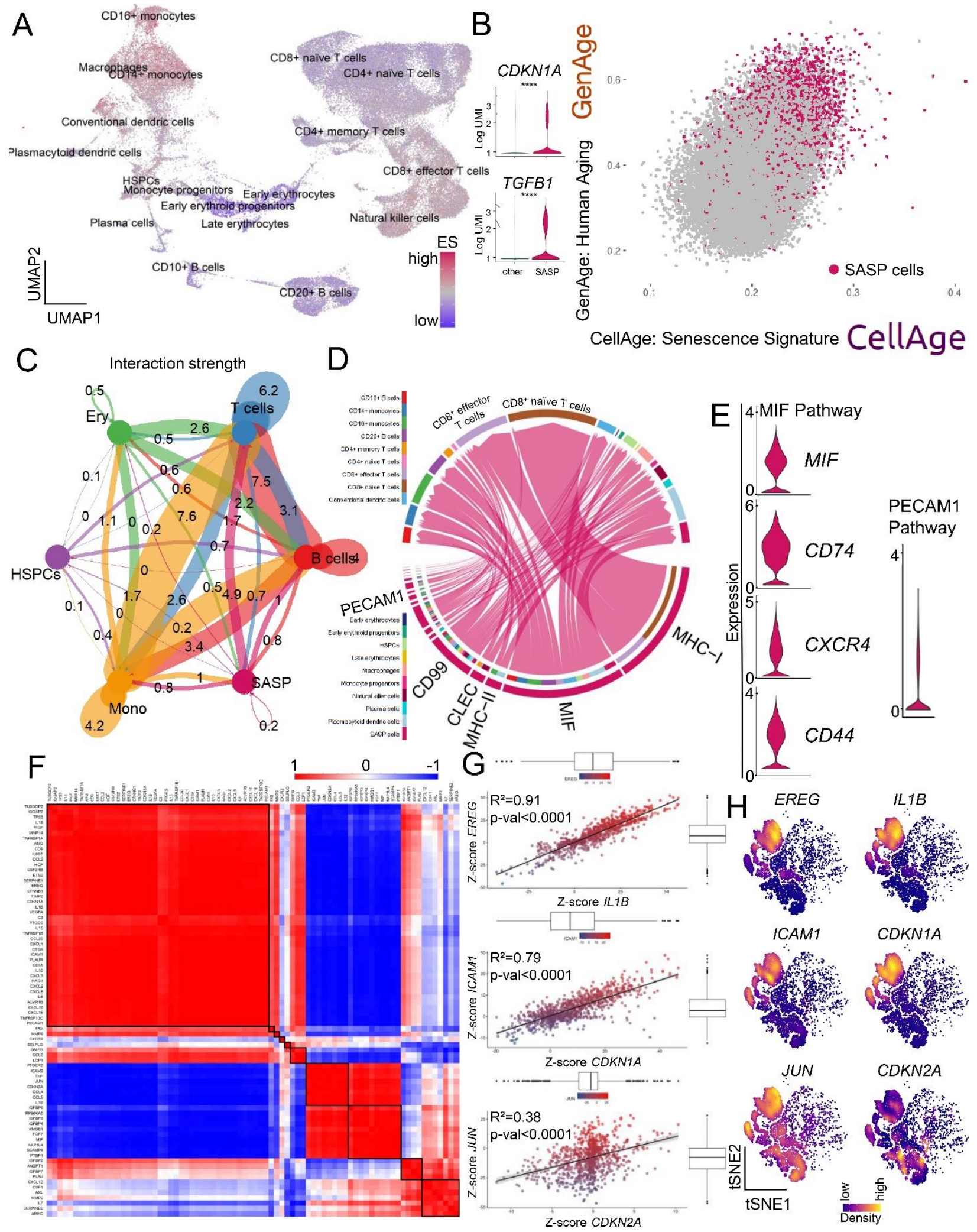

Figure 4. SASP-associated hematopoietic cells in human bone marrow are mainly of monocytic origin and communicate via the MIF pathway. (A) Using a previously published scRNA-seq dataset from human bone marrow (GSE120446, ${ }^{46} \mathrm{n}=68,478$ cells), we performed GSEA at the 
single cell level to uncover cells responsible for senescence/SASP-associated gene expression. The highest enrichment score (ES) for the SenMayo gene set occurred within the $\mathrm{CD}_{14}{ }^{+}$and $\mathrm{CD}_{16}{ }^{+}$monocytic cell cluster, represented in a Uniform Manifold Approximation and Projection (UMAP). We selected the top $10 \%$ of senescence/SASP-expressing cells to form the "SASP cells" $(n=6,850$ cells) cluster displaying a $(B)$ independent enrichment of canonical senescence genes including CDKN1A/p21 ${ }^{C I P 1}$ and TGFB1 and which was likewise enriched for two aging signatures (GenAge: genes associated with aging in model organisms; ${ }^{47}$ and CellAge: positively regulated genes associated with aging in human cells; (C) The SASP cells showed the highest interaction strength with T cells in the bone marrow; (D) Among the interaction targets of SASP cells, T cells were predominantly targeted via the MHC-I, MIF, and PECAM1 pathways; (E) Members of the MIF and PECAM1 signaling pathways showed high expression patterns within the SASP population; (F) SASP cells were characterized by distinct co-expression patterns predicting (functional) clusters (e.g., JUN and CDKN2A), potentially overcoming difficulties of low expression of specific senescence-associated genes such as CDKN2A/P16 ink4A. These strong indicators of co-expression were mathematically isolated by z-scores $(G)$ and spatially summarized $(H)$ in subcell populations within the SASP cluster, as indicated by kernel gene-weighted density estimation in a t-distributed Stochastic Neighbor Embedding (tSNE) representation. ${ }^{\star \star \star *} \mathrm{p}<0.0001, \mathrm{n}=22(10$ $\hat{0}, 12$ +).

This analysis detected multiple cellular clusters that were more highly enriched than others for senescence/SASP genes, i.e., had higher enrichment scores (ES). These high ES clusters included $\mathrm{CD}^{+} 4^{+}$and $\mathrm{CD}^{+} 6^{+}$monocytes as well as macrophages (Fig. 4A, Suppl. Fig. 2A). By selecting the top $10 \%$ of cells with the highest expression of senescence/SASP-associated genes, we generated a new cluster of cells, consisting of 6,850 cells, predominantly of monocytic origin (referred to as "SASP cells" in Fig. 4B). These SASP cells showed an increase in canonical markers of senescence such as CDKN1A/p21 Cip1 and TGFB1, which are independent and not included in the SenMayo gene set, as well as enrichment of previously published gene sets indicative of human ${ }^{47}$ and cellular aging ${ }^{48}$ (Table 3). Visually, the SASP cells had a high correlation with genes in two established aging gene sets (GenAge and positively regulated in CellAge, Fig. 4B). To further elucidate the replicative state of these cells, we compared their cell cycle state with the other clusters. A shift towards the G1 phase occurred within the SASP cells (Suppl. Fig. S2B), consistent with replicative arrest. This finding was supported by cell cycle arrest gene enrichment within the SASP cells (Suppl. Fig. S2C). In addition, pseudotime analysis (Suppl. Fig. S2D, left panel), which permits elucidation of the temporal gene expression pattern of a specific 
cell type, revealed an increase in SASP cells over time (representing differentiation), particularly

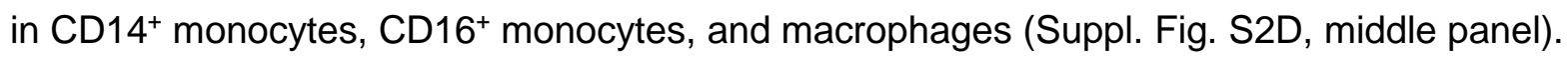

In addition to intracellular signaling pathways differentially regulated in SASP-secreting cells, these cells have been demonstrated to affect surrounding cells. ${ }^{13,49}$ To explore these intercellular interactions, we evaluated potential ligand-receptor interactions and secretion patterns based on underlying gene expression levels in different hematopoietic cell types in human bone marrow. ${ }^{50}$ The strongest interaction of SASP cells was found with T cells, followed by monocytic cells and B cells (Fig. 4C). Among the affected pathways, the major histocompatibility complex class I (MHC-I), Macrophage Migration Inhibitory Factor (MIF), and Platelet And Endothelial Cell Adhesion Molecule 1 (PECAM1, CD31) pathways were most highly enriched (Fig. 4D, E). Of note, in the pseudotime analysis described above, MIF expression also increased markedly in terminally differentiated $\mathrm{CD}_{14}{ }^{+}$and $\mathrm{CD} 16^{+}$monocytes and macrophages and SASP cells (Suppl. Fig. S2D, right panel). Moreover, MIF pathway members including CD74, CXCR4, and CD44 had overall high expression in SASP cells (Fig. 4E). Compared to other cell types, the overall outgoing interaction strength of SASP cells was remarkably high (Suppl. Fig. S3A). Besides their importance as senders, mediators, and influencers (defined by signalling network analysis using centrality measures; for details see, ${ }^{50,51}$ Suppl. Fig. S3B, C), SASP cells displayed a substantial incoming signaling pattern dominated by the MIF, ANNEXIN, CD45, IGGB2, MHC-I, MHC-II, and PECAM1 pathways (Suppl. Fig. S3D). Within these SASP cells, the strongest direct receptor-ligand MIF interaction between the ligand $\mathrm{CD}^{+} 4^{+}$and the receptor CD44 was mainly detected in other monocytic cells, while the MIF interaction via the ligand CD74+receptor CXCR4 pair was significant for SASP to $\mathrm{CD} 10^{+} \mathrm{B}$ and $\mathrm{CD} 20^{+} \mathrm{B}$ cells as well as plasmacytoid dendric cells. The PECAM1 pathway targeted plasma cells and CD16 ${ }^{+}$monocytes (Suppl. Fig. S3E).

Further analysis revealed that the SASP cells were characterized by distinct patterns of co-expression out of which several markers were found to be strongly associated with each other 
(Fig. 4F-G) - e.g., EREG/IL1B, ICAM1/CDKN1A, and JUN/CDKN2A. Out of the 125 genes within the SenMayo panel, some were consistently upregulated (red in Fig. 4F), while others were simultaneously downregulated (blue in Fig. 4F). After we found that some of the "canonical" SASP markers such as EREG/IL1B and SASP/senescence markers such as ICAM1/CDKN1A showed high concordance in their cell-wise expression patterns, we aimed to find surrogate genes for certain low-expressed genes - e.g. CDKN2A/p16 ${ }^{\operatorname{lnk} 4 a}$. Within the SASP cluster, we found a strong correlation between $J U N$ and $C D K N 2 A / p 16^{\text {Ink4a }}$ expression, which represents a potential approach to overcome the challenge of low CDKN2A/p16 $6^{\operatorname{lnk} 4 a}$ expression in sequencing datasets. To independently confirm these correlations, we depicted these genes in a pairwise fashion with kernel density estimation within the SASP cell clusters (Fig. 4H), where the overall SASP cells are in blue and the red/yellow colors indicate higher levels of expression within the SASP cells of each gene. ${ }^{45}$ These analyses thus demonstrate the validity of the SenMayo gene set in a human bone marrow scRNA-seq dataset and identify monocytic cells as the hematopoietic cell population with the highest proportion of SASP-associated cells.

To further test SenMayo in single cell datasets and potentially contrast bone marrow hematopoietic cells to bone/bone marrow mesenchymal cells, we next evaluated a published murine dataset that contained scRNA-seq data from bone and bone marrow mesenchymal cells (GSE128423, ${ }^{52}$ Fig. 5A, n=35,368 cells). We detected a heterogenous distribution of highly enriched cells for SenMayo ("SASP cells", n=3,537), which likewise were enriched in both GenAge and CellAge (Fig. 5B), canonical markers of senescence (Cdkn1a/p21 ${ }^{\text {Cip1 }}$ and Tgfb1, Fig. 5B) and was primarily comprised of cells from the osteolineage (OLC1 and 2) as well as leptin receptor-positive (Lepr+) MSC cluster (Suppl. Fig. S4A shows the fraction of the original clusters that were subsequently assigned to the newly created SASP cluster and Suppl. Fig. S4B indicates the percentage of cells within each cluster that were in the top $10 \%$ of cells enriched for SenMayo genes). Interestingly, $21 \%$ of osteolineage cells (24\% in OLC 1 and $18 \%$ in OLC2) had the highest enrichment for SASP factors (Suppl. Fig. S4B, Table 2). Similar to the human hematopoietic bone 
marrow dataset, murine bone/bone marrow mesenchymal SASP cells displayed a shift in cell cycle phase to the G1 phase (Suppl. Fig. S4C). This was confirmed by gene ontology analysis revealing enrichment of senescence- and cell cycle arrest-associated genes in these cell clusters (Suppl. Fig. 4D). The murine mesenchymal SASP cells were characterized by a high interaction with osteolineage and chondrocytic cells (Fig. 5C), with the MIF and PECAM1 pathways again among those significantly enriched, where these cells mostly acted as senders and influencers (Fig. 5D, Suppl. Fig. S4E). Notably, SASP cells had one of the highest outgoing interaction strengths (Suppl. Fig. S4F). A direct communication of these mesenchymal SASP cells mostly appeared in the MIF pathway (via L/R Mif/Ackr3) with chondrocytic cells and mineralizing osteocytes (Suppl. Fig. S4G). 

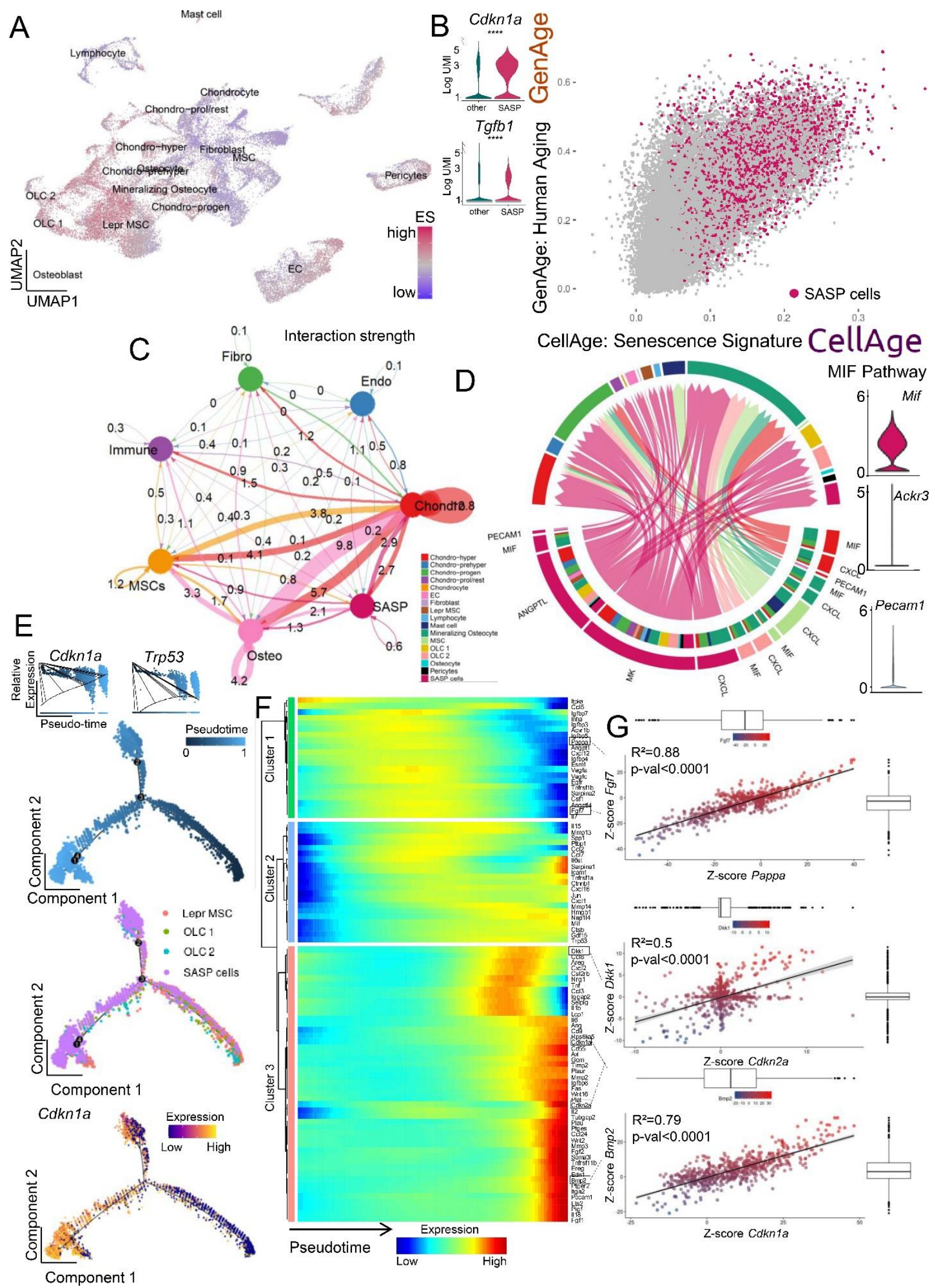
Figure 5. In murine bone and bone marrow mesenchymal cells, osteolineage cells constitute the largest proportion of SASP cells and communicate with osteolineage and chondrocytic cells via the MIF and PECAM1 pathways and show characteristics of terminal differentiation. (A) We analyzed a publicly available murine bone and bone marrow gene set (GSE12842352), and enriched 35,368 cells for the newly created SenMayo gene set; (B) The top 10\% senescence/SASP gene-expressing cells $(n=3,537)$ were assigned to the newly created "SASP cells" cluster. They displayed an increase in canonical markers of senescence including Cdkn1a/p21 $1^{\text {Cip } 1}$ and Tgfb1, and were enriched in the GenAge and CellAge gene sets (GenAge, CellAge ${ }^{47}$ ); (C) The strongest interaction of the SASP cells was narrowed down to chondrocytic cells, while the osteolineage cells were another important crosstalk neighbor; (D) Outgoing interaction patterns of SASP cells (pink, left bottom quarter) indicated the importance of several signaling pathways that resulted in a significant enrichment of Mk, Angptl, Mif and Pecam1; (E) In pseudotime, the SASP cluster was most abundant in the terminal branches, and overexpressed Cdkn1a/p2 $1^{\text {Cip } 1}$ in terminal states (top-left inlay, bottom red color on the left, terminal branch); (F) In their terminal differentiation, the SASP cluster was enriched in several factors, out of which distinct co-expressional patterns were extracted; $(G)$ While the terminal differentiation was marked by a simultaneous loss of Pappa and Fgf7 (cluster 1, green in F), a significant correlation of $D k k 1$ with $C d k n 2 a / p 16^{\text {Ink41 }}$, likewise Bmp2 and $C d k n 1 a / p 21^{C i p 1}$, was mathematically predicted (cluster 2, pink in $\mathrm{F}$ ). ${ }^{* * * *} \mathrm{p}<0.0001, \mathrm{n}=8$ (4 bone, 4 bone marrow, all $\left.{ }^{\Uparrow}\right)$.

The three main origins for the SASP cluster (namely Lepr+ MSCs, OLC 1, and OLC 2), as depicted in pseudotime, demonstrated that the SASP cells accumulated in a terminal developmental branch, coinciding with increased Cdkn1a/p21 ${ }^{\text {Cip1 }}$ and Trp53 expression (Fig. 5E). Further analysis of these pseudotime expression patterns showed that certain genes followed defined modules (green, blue, and red in Fig. 5F), which then formed co-expressional patterns (Suppl. Fig. S5A). Within the SASP cluster, these co-expressional patterns could be imaged at an individual cell level, predicting genes of similar abundance within some cells (Fig. 5G). For example, while Pappa and Fgf7 were simultaneously downregulated in terminally differentiated stages (Fig. 5F, blue color in the green cluster, Fig. 5G top), they were part of a modular cluster (Suppl. Fig. S5A, black boxes on the left, fifth square from above). We also performed kernelweighed density estimation (Suppl. Fig. S5B), confirming our results that Fgf7 and Pappa were co-expressed in the SASP cells. Likewise, Dkk1 and Cdkn2a/p16 $6^{\operatorname{lnk} 4 a}$ displayed the mathematically predicted comparable expression patterns in kernel-weighed density, displayed in tSNE, as did Bmp2 and Cdkn1a/p21 ${ }^{\text {Cip1 }}$ (Fig. 5G, Suppl. Fig. S5B). 
Further experimental validation of in silico analyses. The above analyses of both hematopoietic and mesenchymal scRNA-seq data pointed to Mif as a key SASP gene that should increase with senescent cell burden and be reduced following clearance of senescent cells. Thus, as a final validation of our in silico analyses, we examined Mif mRNA levels by RT-qPCR in our mouse models and found that as predicted, Mif mRNA levels were increased in the bones from old compared to young mice (Fig. 6A) and were significantly reduced following the genetic clearance of senescent cells with AP in old INK-ATTAC mice (Fig. 6B).
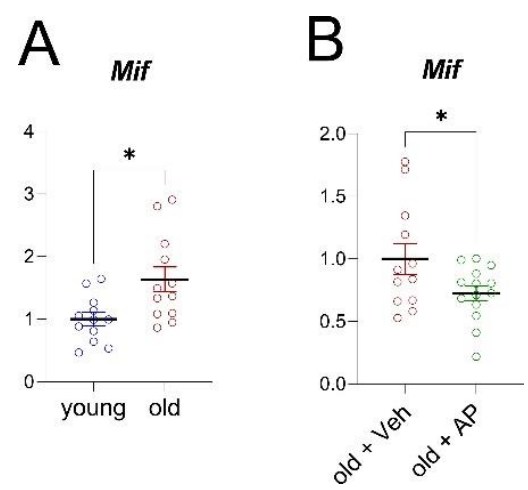

Figure 6. The in silico predicted importance of the Mif pathway is reflected in the aged INK-ATTAC mouse model. (A) We compared young $(n=12)$ and old vehicle-treated mice $(n=13)$, and old mice treated with AP $(n=16)$. (A) Upregulation of Mif was confirmed by RT-qPCR ( $n=24$ young (12 Veh, 12 old (all + )); (B) The clearance of senescent cells in the aged cohort by AP treatment reduced this Mif expression ( $n=26$ old (12 Veh, 14 AP (all +$)) .^{*} p<0.05$ 


\section{Discussion}

The identification and characterization of senescent cells, particularly in bulk or scRNAseq data, has been problematic for a number of reasons, including variable detection of low levels of the $C d k n 2 a / p 16^{\operatorname{lnk} 4 a}$ transcript even in senescent cells ${ }^{45}$ and the lack of a consistent gene panel that can reliably identify these cells. Thus, we generated a gene set (SenMayo) consisting of 125 previously identified senescence/SASP-associated factors and first validated it in bone biopsy samples from two human cohorts consisting of young vs elderly women. ${ }^{27,28}$ Importantly, to establish this as a senescence, rather than just "aging" gene set, we demonstrated that clearance of senescent cells in mice and in humans resulted in significant reductions of SenMayo. Using publicly available RNA-seq data, we demonstrated applicability across tissues and species and also found that SenMayo performed better than six existing senescence/SASP gene panels. ${ }^{14,40-}$ ${ }^{44}$ We next applied SenMayo to publicly available bone marrow/bone scRNA-seq data and successfully characterized hematopoietic and mesenchymal cells expressing high levels of senescence/SASP markers at the single cell level, demonstrated co-expression (where feasible) with the key senescence genes, Cdkn2a/p16 ${ }^{\text {Ink4a }}$ and $C d k n 1 a / p 21^{C i p 1}$, and analyzed intercellular communication patterns of senescent cells with other cells in their microenvironment. Based on these analyses, we found that senescent hematopoietic and mesenchymal cells communicated with other cells through common pathways, including the Macrophage Migration Inhibitory Factor (MIF) pathway, which has been implicated not only in inflammation but also in immune evasion, an important property of senescent cells. ${ }^{53}$ Finally, as a key validation of our in silico analyses, we then examined Mif mRNA levels by RT-qPCR in our mouse models and found that as predicted, Mif mRNA levels were increased in bones from old compared to young mice and were significantly reduced following the genetic clearance of senescent cells in the old mice.

The heterogeneous composition of the SASP, which consists of a multitude of growth factors, chemokines, cytokines, and matrix-degrading proteins, has been experimentally verified using various in vitro systems to induce cell stress, in vivo using multiple pre-clinical animal 
models of aging and disease, and has been linked to several pathophysiological conditions in humans as well as clinical outcomes. ${ }^{54,55}$ In the current study, we were able to group these factors into 9 distinct clusters to form tightly connected networks with distinct key molecules. The importance of these and other SASP factors has been verified in multiple biological contexts. ${ }^{56-63}$ Interestingly, the control of the SASP itself by RELA/p65, which we detected in two sequencing datasets of aging women, has recently been experimentally verified in U2OS osteosarcoma cells. ${ }^{64}$

Transcriptome-wide state-of-the-art technologies such as scRNA-seq will help shape our understanding of not just aging, but also therapeutics that potentially target fundamental mechanisms of aging, such as senolytics. As noted earlier, a confounder in these analyses is the generally low expression of the canonical marker of senescence, Cdkn2a/p16 ${ }^{\operatorname{lnk} 4 a}$, which is clearly detectable by RT-qPCR in the setting of aging, but poses challenges when using transcriptomewide approaches. ${ }^{45}$ Hence, we propose a species-specific co-expression analysis with JUN (Homo sapiens) or Dkk1 (Mus musculus), based on modules of comparable expression to address this challenge. To our knowledge, we for the first time leveraged publicly available single cell datasets to enrich for a senescence/SASP gene set. Since we did not include commonly used senescence-markers $\left(C d k n 2 a / p 16^{\text {Ink4a }}, C d k n 1 a / p 21^{\text {Cip } 1}\right)$ in the SenMayo panel, we were still able to rely on them to confirm a senescent cell state. Additional verification included a shift in the cell cycle phase to $\mathrm{G} 1$, as senescence prevents cells from proceeding to the $\mathrm{S}$ or $\mathrm{M}$ phases. ${ }^{59,65,66}$ With Cdkn1a/p21 ${ }^{\text {Cip1 }}$ being expressed at relatively higher levels, we were able to verify a senescent status of SASP cells, confirming our approach to identify single cells expressing high levels of SenMayo genes as being senescent.

The use of pseudotime in scRNA-seq datasets to predict age-associated changes and fate commitment has been demonstrated previously in muscle stem cells (MuSCs) and fibroadipose progenitors (FAPs) ${ }^{67}$ These analyses pointed to the importance of TGF- $\beta$ signaling, but without specifically focusing on age-related expression changes. By contrast, we used 
pseudotime analyses to establish a novel approach to identify age-dependent transcriptional changes in senescence/SASP genes distinct from Cdkn2a/p16 ${ }^{\text {Ink4a }}$ and Cdkn1a/p21 Cip1.

Using a z-score based probabilistic model with pairwise correlations (bigSCale ${ }^{68}$ ) to construct transcriptional networks, several groups have successfully established the use of withincell networks in single cell datasets ${ }^{25,69}$ and we made use of this approach to define senescence modules of similar expression. With overall agreement between pseudotime, network analyses, and direct pairwise z-score prediction, we overcame the downside of normalized expression, and a z-score predicted space allowed us to assign clusters and spatially depict them within cellular aggregates. These modules may serve as sources for novel senescent markers and pathways. ${ }^{70}$

As noted earlier, the MIF pathway emerged as a key intercellular communication pathway used by both hematopoietic and mesenchymal cells in bone marrow expressing high levels of senescence/SASP genes. This is perhaps not surprising given the importance of MIF as a proinflammatory cytokine, inhibitor of $\mathrm{p} 53$, and positive regulator of NF-kB. ${ }^{71}$ MIF appears to be pivotal for cellular senescence, aging, and joint inflammation; however, its presence has been associated with a beneficial effect on the healthy lung and in MSCs. ${ }^{72-77}$ Of note, recent evidence indicates an important role for MIF signaling in immune evasion by tumors ${ }^{78}$ and parasites, ${ }^{79}$ raising the possibility that increased MIF expression by multiple senescent cell types may play a role in the ability of senescent cells to resist immune clearance, particularly with aging, ${ }^{53}$ and this possibility warrants further study. Importantly, we also used Mif expression to validate our in silico predictions based on the scRNA-seq analyses, and confirmed both an increase in Mif expression with aging in murine bone as well as a reduction in Mif mRNA levels following genetic clearance of senescent cells.

The development and validation of SenMayo, as demonstrated here, may be particularly timely in the context of the recent establishment of a major NIH Common Fund consortium to map senescent cells (SenNET, https://sennetconsortium.org/). The goal of this program is to "comprehensively identify and characterize the differences in senescent cells across the body, 
across various states of human health, and across the lifespan." The application of SenMayo to bulk or scRNA-seq data from SenNET should greatly facilitate this goal and could provide a standardized gene set that is used across the multiple sites involved in this consortium.

In summary, our studies contribute a novel gene set (SenMayo) that increases with aging across tissues and species, is responsive to senescent cell clearance, and can be used both in bulk and scRNA-seq analyses to identify cells expressing high levels of senescence/SASP genes. This gene set also has potential utility in the clinical evaluation of senescent cell burden and for studies of senolytic therapies. In addition, SenMayo circumvents current limitations in the transcriptional identification of senescent cells at the single cell level, thereby allowing for detailed analyses (e.g. pseudotime, intercellular signaling) that will facilitate better characterization of these cells in future studies. 


\section{Methods}

\section{MATERIALS AND METHODS}

Generation of SenMayo. Our own GSEA gene set for senescence-associated genes was generated by combining genes that had been reported in previous studies to be enriched in senescent and/or SASP-secreting cells and experimentally verified in at least human or mouse cells. We screened 1,656 studies, but following removal of studies reporting duplicates, case reports, and non-human or non-murine genes, formulated a list of 15 studies from which we identified 125 genes that constituted SenMayo (Table $1^{18,26,48,55,61,80-89}$ ). Note that we intentionally did not include $C D K N 2 A / p 16^{\text {Ink }}$ or $C D K N 1 A / p 21^{\text {Cip1 }}$ in SenMayo as we used these genes, in part, to validate our senescence/SASP gene set.

$R N A$-seq. Transcriptome-wide gene expression data from young $(\mathrm{n}=15,30.9 \pm 4.0$ years and $\mathrm{n}=19$, $30.3 \pm 5.4$ years) and postmenopausal females $(n=15,68.7 \pm 4.8$ years and $n=19,73.1 \pm 6.6$ years) as well as 10 diabetic kidney disease patients ( 8 male, 2 female, $71.25 \pm 7.85$ years and $65.0 \pm 8.0$ years, respectively) were analyzed from three previous studies performed by our group (GSE141595: NCT02554695, GSE72815: NCT02349113, ${ }^{24,38: ~ N C T 02848131) 27,28 . ~ A f t e r ~ t h e ~}$ original interventional study in diabetic kidney disease patients was completed, one additional female patient was added. All human studies were approved by the Mayo Clinic Institutional Review Board and written informed consent was obtained from all participants. RNA was isolated from whole bone biopsies (which included bone and bone marrow cells, Cohort $A)^{27}$ as well as bone biopsies that were processed to remove bone marrow and bone surface cells and were thus highly enriched for osteocytes (Cohort B), ${ }^{28}$ and adipose tissue, $2-5 \mathrm{~cm}$ inferior to the navel (for details, $\left.\operatorname{se}^{38}\right)$. Subcutaneous adipose tissue was obtained by an elliptical incisional biopsy at a point to the right or left, and $2-5 \mathrm{~cm}$ inferior to the navel. ${ }^{24,38}$ Sequencing was performed on a HiSeq2000 (Illumina ${ }^{\circledR}$ ), fastq files were mapped to the human reference genome hg19, and analysis was performed as previously described. ${ }^{27,28}$ Significantly differentially regulated genes 
were selected by a Benjamini-Hochberg adjusted $p$-value $<0.05$ and $\log _{2}$-fold changes above 0.5 or below -0.5. Gene Set Enrichment Analysis $\left(\mathrm{GSEA}^{31,90}\right)$ was performed with default settings (1000 permutations for gene sets, Signal2Noise metric for ranking genes). The network analysis was conducted with Cytoscape 3.8.2. ${ }^{30}$ For mRNA-seq of murine material, tibiae were centrifuged as noted above to remove bone marrow elements and then were immediately homogenized in QIAzol Lysis Reagent (QIAGEN, Valencia, CA) and stored at $-80^{\circ} \mathrm{C}$, until the time of RNA extraction. RNA-sequencing was performed on a HiSeq2000 (Illumina ${ }^{\circledR}$ ), fastq files were mapped to the murine reference genome $\mathrm{mm} 10$, and analysis was performed as previously described. ${ }^{27,28}$ An example of the code used for RNA-seq can be found in the provided R notebook (Methods: GSE72815_YOE_Notebook.Rmd).

Mouse strains and drug treatments. All animal protocols were approved by the Institutional Animal Care and Use Committee (IACUC), and all experiments were performed in accordance with IACUC guidelines. Mice were housed in ventilated cages in a pathogen-free facility (12-hour light/dark cycle, $23^{\circ} \mathrm{C}$ ) and had access to food (standard mouse diet, Lab Diet 5053, St. Louis, MO) and water ad libitum. Mouse experiments for a genetic targeting approach of senescent cells have been described by our group earlier. ${ }^{37}$ Briefly, old (20 months) female mice were injected intraperitoneally with vehicle (4\% of $100 \% \mathrm{EtOH}, 10 \% \mathrm{PEG} 400,86 \%$ of $2 \%$ Tween 20 in deionized Water) or AP20187 (B/B homodimerizer, Clontech; $10 \mathrm{mg}$ of AP20187 per kg body mass) twice weekly at the age of 20 months for a total of 4 months (old mice were sacrificed at 24 months of age). In addition, young (6-month) INK-ATTAC mice were used as a control comparison cohort.

Quantitative real-time polymerase chain reaction ( $R T$-qPCR) analysis. For bone analyses, tibiae were centrifuged to remove marrow elements and then immediately homogenized in QIAzol Lysis Reagent (QIAGEN, Valencia, CA) and stored at $-80^{\circ} \mathrm{C}$. Subsequent RNA extraction, cDNA synthesis, and targeted gene expression measurements of mRNA levels by RT-qPCR were 
performed as described previously. ${ }^{91}$ Total RNA was extracted according to the manufacturer's instructions using QIAzol Lysis Reagent. Purification with RNeasy Mini Columns (QIAGEN, Valencia, CA) was subsequently performed. On-column RNase-free DNase solution (QIAGEN, Valencia, CA) was applied to degrade contaminating genomic DNA. RNA quantity was assessed with Nanodrop spectrophotometry (Thermo Fisher Scientific, Wilmington, DE). Standard reverse transcriptase was performed using High-Capacity cDNA Reverse Transcription Kit (Applied Biosystems by Life Technologies, Foster City, CA). Transcript mRNA levels were determined by RT-qPCR on the ABI Prism 7900HT Real Time System (Applied Biosystems, Carlsbad, CA) using SYBR green (Qiagen, Valencia, CA). The mouse forward primer sequence (5'-3') for Mif was: 5'GCCACCATGCCTATGTTCATC-3' and Reverse Primer Sequence 5'GGGTGAGCTCCGACAGAAAC-3'. RNA was normalized using two reference genes (Actb [forward: 5'-AATCGTGCGTGACATCAAAGAG-3', reverse: 5'-GCCATCTCCTGCTCGAAGTC3'], Gapdh [forward: 5'-GACCTGACCTGCCGTCTAGAAA-3', reverse: 5'CCTGCTTCACCACCTTCTTGA-3']) from which the most stable housekeeping gene was determined by the geNorm algorithm. For each sample, the median cycle threshold (Ct) of each gene (run in triplicate) was normalized to the geometric mean of the median $\mathrm{Ct}$ of the most stable reference gene. The delta Ct for each gene was used to calculate the relative mRNA expression changes for each sample. Genes with $\mathrm{Ct}$ values $>35$ were considered not expressed (NE), as done previously. ${ }^{92}$

Single-cell RNA-seq (scRNA-seq) analysis. Transcriptome-wide analysis of human bone marrow mononuclear cells at a single cell level was based on a previously published dataset. ${ }^{46}$ Here, bone marrow was isolated from healthy female $(n=10)$ and male $(n=10)$ donors $(50.6 \pm 14.9$ years $)$ and droplet-based scRNA-seq was performed. A minimum sequencing depth of 50,000 reads/cell with a mean of 880 genes/cell was reported. In addition, we analyzed droplet-based scRNA-seq data from bone marrow cells isolated from C57BL/6 mice $(n=14)^{52}$ and from C57BL/6JN mice $(n=30)^{35}$ 
and from the tabula muris senis. ${ }^{35}$ Sequencing data were aligned to the human reference genome Grch38 and the mouse genome mm10, respectively. Data with at least 500 unique molecular identifiers (UMIs), log10 genes per UMI >0.8, >250 genes per cell and a mitochondrial ratio of less than $20 \%$ were extracted, normalized, and integrated using the Seurat package v3.0 in R4.0.2. Subsequent R-packages were Nebulosa $\left(3.13^{93}\right)$, Monocle (2.18.094), dittoSeq (1.2.6 $\left.6^{95}\right)$, Escape (1.0.1, "Borcherding N, Andrews J (2021). escape: Easy single cell analysis platform for enrichment. R package version 1.2.0."), Cellchat $^{50}$ (within the Cellchat package, and for Fig. 2C, "CD10+ B cells", "CD20+ B cells", "Plasma cells", "Plasmacytoid dendric cells", "Conventional dendric cells" were summarized as "B cells", "CD4+ naïve T cells", "CD4+ memory T cells", "CD8+ naïve T cells", "CD8+ effector T cells" were summarized as "T cells", "Early erythroid progenitors", "Early erythrocytes", "Late erythrocytes" as "Ery”, "HSPCs” as "HSPCs”, "Monocyte progenitors", "CD14+ monocytes", "CD16+ monocytes", "Macrophages", "Natural killer cells" as "Mono" and "SASP cells" as "SASP". For Figure 3C, "Chondro-hyper", "Chondro-prehyper", "Chondroprogen", "Chondro-prol/rest", "Chondrocyte" were summarized as "Chondro", "EC", "Pericytes" as "Endo", "Fibroblast" as "Fibro", "Lymphocyte", "Mast cell" as "Immune", "Lepr MSC", "MSC" as "MSC", "Mineralizing Osteocyte", "OLC 1", "OLC 2", "Osteoblast", "Osteocyte" as "Osteo" and "SASP cells" as “SASP”), bigSCale $\left(2.1^{70}\right)$, gprofiler2 $\left(0.2 .0^{96}\right)$, igraph (1.2.6, Csardi G, Nepusz T (2006). "The igraph software package for complex network research." InterJournal, Complex Systems, 1695), PCAtools (2.4.0, Blighe K, Lun A (2021). PCAtools: PCAtools: Everything Principal Components Analysis. R package version 2.4.0), and corrplot (0.89).

Pseudotime is a progression of cells along a virtually estimated path, mimicking temporal development. By using Monocle, an independent component analysis (ICA) dimensional reduction is performed, followed by a projection of a minimal spanning tree (MST) of the cell's location in this reduced space. Each cell is assigned a pseudotemporal space. ${ }^{97,98}$ Monocle 2 was used to preprocess, perform UMAP reduction, and reduce the dimensionality using the DDRTree algorithm with a maximum of four dimensions. Subsequently, the cells were ordered and genes 
plotted along the reduced dimension. Differential gene testing has been performed with the formula " sm.ns(Pseudotime)", and the results were restricted by a qvalue $<0.1 .^{97}$

An example of the code used for scRNA-seq can be found in the provided R notebook (Methods: R_notebook_Fig4_5_sup2to5.Rmd).

Author contributions. D.S., J.N.F., and S.K. conceived and directed the project. D.S. and J.N.F. designed the experiments and interpreted the data with input from S.K. Experiments were performed by D.S. and R.L.K. D.S. and S.K. wrote the manuscript. All authors reviewed the manuscript. J.N.F. and S.K. oversaw all experimental design, data analyses, and manuscript preparation. J.N.F., S.K., and D.S. accept responsibility for the integrity of the data analysis.

Acknowledgements. This work was supported by the German Research Foundation (D.F.G., 413501650) (D.S.), National Institutes of Health (NIH) grants P01 AG062413 (S.K., J.N.F., N.K.L., R.P., P.D.R., L.J.N., Y.I., J.P., D.G.M., T.T., J.L.K.), R21 AG065868 (S.K., J.N.F), K01 AR070241 (J.N.F.), R01 AG063707 (D.G.M.), R37 AG 013925 (J.L.K., T.T.), R33AG 61456 (J.L.K., T.T., R.P., P.D.R., L.J.N., S.K.), 1R01AG068048-01 (JFP), R56 AG 60907 and R01 AG55529 (N.K.L.)., the Connor Fund (J.L.K., T.T.), Robert P. and Arlene R. Kogod (J.L.K.), Robert J. and Theresa W. Ryan (J.L.K., T.T.), the Noaber Foundation (J.L.K., T.T.), and Mildred Scheel postdoc fellowship by the German Cancer Aid (R.L.K.). X.Z. is supported by the Robert and Arlene Kogod Center on Aging Career Development Award.

The authors thank SA Johnsen and FH Hamdan for inspiring discussions.

Competing interests. Patents on senolytic drugs and their uses and SASP biomarkers are held by Mayo Clinic and the University of Minnesota. This research has been reviewed by the Mayo Clinic Conflict of Interest Review Board and was conducted in compliance with Mayo Clinic Conflict of Interest policies. 
bioRxiv preprint doi: https://doi.org/10.1101/2021.12.10.472095; this version posted December 11, 2021. The copyright holder for this preprint

(which was not certified by peer review) is the author/funder. All rights reserved. No reuse allowed without permission.

Table 1. Genes included in the SenMayo panel. The relationship of each gene to senescence/aging is described in the reference indicated.

\begin{tabular}{|c|c|c|c|}
\hline Gene(human) & Classification & State & Reference \\
\hline$A C V R 1 B$ & Transmembrane sianal receptors & Transmembrane & 26 \\
\hline$A N G$ & Miscellaneous & Secreted & 80,87 \\
\hline ANGPT1 & Intercellular signal molecule & Secreted & 55 \\
\hline ANGPTL4 & Intercellular signal molecule & Secreted & 18,55 \\
\hline$A R E G$ & Growth factor & Intracellular & $80,87,88$ \\
\hline$A X L$ & Transmembrane signal receptors & Transmembrane & 18,88 \\
\hline$B E X 3$ & Miscellaneous & Intracellular & 18 \\
\hline BMP2 & Growth factor & Secreted & 55,88 \\
\hline BMP6 & Growth factor & Secreted & 88 \\
\hline C3 & Protease inhibitors & Secreted & 55 \\
\hline CCL1 & Cytokine/Chemokine & Secreted & 88 \\
\hline CCL13 & Cytokine/Chemokine & Secreted & 80,88 \\
\hline CCL16 & Cytokine/Chemokine & Secreted & $26,80,88$ \\
\hline CCL2 & Cytokine/Chemokine & Secreted & $26,55,82,83,85,87,88$ \\
\hline CCL2O & Cytokine/Chemokine & Secreted & $26,80,82$ \\
\hline CCL24 & Cytokine/Chemokine & Secreted & 87 \\
\hline CCL26 & Cytokine/Chemokine & Secreted & 80,88 \\
\hline $\mathrm{CCL} 3$ & Cytokine/Chemokine & Secreted & $55,84,87,88$ \\
\hline CCL3L1 & Cytokine/Chemokine & Secreted & 55 \\
\hline CCL4 & Cytokine/Chemokine & Secreted & 87 \\
\hline CCL5 & Cytokine/Chemokine & Secreted & $26,81,87$ \\
\hline CCL7 & Cytokine/Chemokine & Secreted & 88 \\
\hline CCL8 & Cytokine/Chemokine & Secreted & 80,88 \\
\hline CD55 & Miscellaneous & Secreted & 88 \\
\hline CD9 & Transmembrane signal receptors & Transmembrane & 18,88 \\
\hline CSF1 & Cytokine/Chemokine & Secreted & 83 \\
\hline CSF2 & Cytokine/Chemokine & Secreted & $26,55,61,80,87,88$ \\
\hline CSF2RB & Transmembrane signal receptors & Transmembrane & 88 \\
\hline CST4 & Protease inhibitors & Secreted & 84 \\
\hline CTNNB1 & Transcription factors and regulators & Transmembrane & 87 \\
\hline CTSB & (Metallo-)proteases & Secreted & 80 \\
\hline CXCL1 & Cytokine/Chemokine & Secreted & $26,55,61,80,87-89$ \\
\hline CXCL10 & Cytokine/Chemokine & Secreted & $55,81,87$ \\
\hline CXCL12 & Cytokine/Chemokine & Secreted & 80,87 \\
\hline CXCL16 & Cytokine/Chemokine & Secreted & 87 \\
\hline CXCL2 & Cytokine/Chemokine & Secreted & $26,61,80,87$ \\
\hline CXCL3 & Cytokine/Chemokine & Secreted & $55,63,80$ \\
\hline CXCL8 & Cytokine/Chemokine & Secreted & $26,55,61,80,82,89$ \\
\hline CXCR2 & Cytokine/Chemokine & Transmembrane & 26 \\
\hline$D K K 1$ & Intercellular signal molecule & Secreted & 55 \\
\hline$E D N 1$ & Intercellular signal molecule & Secreted & 55 \\
\hline$E G F$ & Transmembrane signal receptors & Transmembrane & 80 \\
\hline$E G F R$ & Transmembrane signal receptors & Transmembrane & 80,88 \\
\hline$E R E G$ & Growth factor & Secreted & $80,87,88$ \\
\hline ESM1 & Intercellular signal molecule & Secreted & 55 \\
\hline ETS2 & Transcription factors and regulators & Intracellular & 88 \\
\hline FAS & Transmembrane signal receptors & Transmembrane & 80,88 \\
\hline FGF1 & Growth factor & Secreted & 26,55 \\
\hline FGF2 & Growth factor & Secreted & 55,80 \\
\hline FGF7 & Growth factor & Secreted & $55,80,88$ \\
\hline GDF15 & Growth factor & Secreted & $26,55,88$ \\
\hline GEM & Miscellaneous & Intracellular & 88 \\
\hline GMFG & Intercellular signal molecule & Intracellular & 88 \\
\hline HGF & (Metallo-)proteases & Secreted & $26,80,87,88$ \\
\hline HMGB1 & Transcription factors and requlators & Intracellular & 55,87 \\
\hline ICAM1 & Miscellaneous & Intracellular & $61,80,87,88$ \\
\hline ICAM3 & Miscellaneous & Intracellular & 87,88 \\
\hline IGF1 & Growth factor & Secreted & $18,81,86,88$ \\
\hline IGFBP1 & Protease inhibitors & Secreted & 88 \\
\hline IGFBP2 & Protease inhibitors & Secreted & $26,61,80,87,88$ \\
\hline IGFBP3 & Protease inhibitors & Secreted & $26,48,55,61,80,87$ \\
\hline IGFBP4 & Protease inhibitors & Secreted & $26,61,80,87$ \\
\hline IGFBP5 & Protease inhibitors & Secreted & $26,55,61,87$ \\
\hline IGFBP6 & Protease inhibitors & Secreted & $26,61,80,87,88$ \\
\hline IGFBP7 & Miscellaneous & Secreted & $26,55,61,80,82,87$ \\
\hline IL10 & Cytokine/Chemokine & Secreted & 85 \\
\hline IL13 & Cytokine/Chemokine & Secreted & 80,88 \\
\hline IL15 & Cytokine/Chemokine & Secreted & $40,83,87,88$ \\
\hline
\end{tabular}


bioRxiv preprint doi: https://doi.org/10.1101/2021.12.10.472095; this version posted December 11, 2021. The copyright holder for this preprint

(which was not certified by peer review) is the author/funder. All rights reserved. No reuse allowed without permission.

\begin{tabular}{|c|c|c|c|}
\hline IL18 & Cytokine/Chemokine & Secreted & 55,87 \\
\hline IL1A & Cytokine/Chemokine & Secreted & $26,40,55,61,80,81,87-89$ \\
\hline IL1B & Cytokine/Chemokine & Secreted & $18,40,85,87-89$ \\
\hline IL2 & Cytokine/Chemokine & Secreted & 87 \\
\hline IL32 & Cytokine/Chemokine & Secreted & 55 \\
\hline IL6 & Cvtokine/Chemokine & Secreted & $26,40,55,61,81-83,85,87,88$ \\
\hline IL6ST & Transmembrane sianal receptors & Transmembrane & 40 \\
\hline IL7 & Cvtokine/Chemokine & Secreted & 40,88 \\
\hline INHA & Growth factor & Secreted & 88 \\
\hline IQGAP2 & Miscellaneous & Intracellular & 88 \\
\hline ITGA2 & Transmembrane sianal receptors & Transmembrane & 88 \\
\hline ITPKA & Protein modifying enzymes & Intracellular & 88 \\
\hline JUN & Transcription factors and requlators & Intracellular & 88 \\
\hline KITLG & Growth factor & Intracellular & 40,87 \\
\hline LCP1 & Miscellaneous & Intracellular & 55 \\
\hline MIF & Protein modifying enzymes & Secreted & $18,40,87,88$ \\
\hline MMP1 & (Metallo-)proteases & Secreted & $40,61,88$ \\
\hline MMP10 & (Metallo-)proteases & Secreted & $40,61,88$ \\
\hline MMP12 & (Metallo-)proteases & Secreted & 40,87 \\
\hline MMP13 & (Metallo-)proteases & Secreted & 40,87 \\
\hline MMP14 & (Metallo-)proteases & Intracellular & 40,87 \\
\hline MMP2 & (Metallo-)proteases & Secreted & 88 \\
\hline MMP3 & (Metallo-)proteases & Secreted & $40,61,84,88$ \\
\hline MMP9 & (Metallo-)proteases & Secreted & 55,84 \\
\hline NAP1L4 & Miscellaneous & Intracellular & 88 \\
\hline$N R G 1$ & Growth factor & Secreted & $40,87,88$ \\
\hline PAPPA & (Metallo-)proteases & Secreted & 55 \\
\hline PECAM1 & Miscellaneous & Intracellular & 88 \\
\hline$P G F$ & Growth factor & Secreted & 87 \\
\hline PIGF & Protein modifying enzymes & Transmembrane & 40,88 \\
\hline PLAT & (Metallo-)proteases & Secreted & 40,87 \\
\hline$P L A U$ & (Metallo-)proteases & Secreted & 40 \\
\hline PLAUR & Transmembrane sianal receptors & Transmembrane & 40,88 \\
\hline PTBP1 & Miscellaneous & Intracellular & 55 \\
\hline PTGER2 & Transmembrane sianal receptors & Transmembrane & 55 \\
\hline PTGES & Protein modifyina enzymes & Intracellular & 88 \\
\hline RPS6KA5 & Protein modifying enzymes & Intracellular & 88 \\
\hline SCAMP4 & Miscellaneous & Intracellular & 55 \\
\hline SELPLG & Transmembrane signal receptors & Transmembrane & 55 \\
\hline SEMA3F & Intercellular sianal molecule & Secreted & 55 \\
\hline SERPINB4 & Protease inhibitors & Intracellular & 55 \\
\hline SERPINE1 & Protease inhibitors & Secreted & $26,40,55,61,82,84,87,88$ \\
\hline SERPINE2 & Protease inhibitors & Secreted & 40,87 \\
\hline SPP1 & Cytokine/Chemokine & Secreted & 55 \\
\hline$S P X$ & Intercellular signal molecule & Secreted & 55 \\
\hline TIMP2 & Protease inhibitors & Secreted & $18,40,87,88$ \\
\hline TNF & Cytokine/Chemokine & Secreted & 81,85 \\
\hline TNFRSF10C & Transmembrane signal receptors & Transmembrane & 40 \\
\hline TNFRSF11B & Transmembrane signal receptors & Transmembrane & 40,88 \\
\hline TNFRSF1A & Transmembrane signal receptors & Transmembrane & 40,87 \\
\hline TNFRSF1B & Transmembrane signal receptors & Transmembrane & 40 \\
\hline TUBGCP2 & Miscellaneous & Intracellular & 88 \\
\hline VEGFA & Growth factor & Secreted & $26,40,82,88$ \\
\hline VEGFC & Growth factor & Secreted & 88 \\
\hline VGF & Intercellular signal molecule & Secreted & 55 \\
\hline WNT16 & Intercellular signal molecule & Secreted & 55 \\
\hline WNT2 & Intercellular sianal molecule & Transmembrane & 88 \\
\hline
\end{tabular}


Table 2. Comparison of SenMayo with 6 existing senescence/SASP gene sets. Note that in GSEA analyses, p-values $<0.25$ are considered potentially significant ${ }^{31,99}$, although we also identified $p$-values $<0.05$ and $<0.01$ (NES, normalized Enrichment Score).

\begin{tabular}{|c|c|c|c|c|c|c|c|c|c|c|c|c|c|c|c|c|c|c|}
\hline & \multicolumn{4}{|c|}{ Human Aging } & \multicolumn{8}{|c|}{ Mouse Aging } & \multicolumn{4}{|c|}{$\begin{array}{c}\text { Mouse Genetic } \\
\text { Clearance of Senescent } \\
\text { Cells } \\
\end{array}$} & \multirow{2}{*}{\multicolumn{2}{|c|}{$\begin{array}{c}\text { Human } \\
\begin{array}{c}\text { Pharmacological } \\
\text { Clearance of } \\
\text { Senescent Cells }\end{array} \\
\text { Adipose (Control vs } \mathrm{D}+\mathrm{Q} \\
\end{array}$}} \\
\hline & \multicolumn{2}{|c|}{ Cohort A } & \multicolumn{2}{|c|}{ Cohort B } & \multicolumn{2}{|c|}{ Microglia } & \multicolumn{2}{|c|}{ Prefrontal cortex } & \multicolumn{2}{|c|}{ Dorsal hippocampus } & \multicolumn{2}{|c|}{ Bone marrow } & \multicolumn{2}{|c|}{$\begin{array}{c}\text { Mouse INK-ATTAC } \\
\text { (old vs young) }\end{array}$} & \multicolumn{2}{|c|}{$\begin{array}{l}\text { Mouse INK-ATTAC } \\
\text { (old, vehicle vs AP) }\end{array}$} & & \\
\hline & NES & $\mathrm{p}$-value & NES & $\mathrm{p}$-value & NES & $\mathrm{p}$-value & NES & $\mathrm{p}$-value & NES & $p$-value & NES & $\mathrm{p}$-value & NES & $p$-value & NES & $\mathrm{p}$-value & NES & $p$-value \\
\hline $\begin{array}{l}\text { R-HSA- } \\
2559582 \\
\end{array}$ & 1.1075 & 0.2826 & 0.6780 & 0.9278 & 1.1794 & 0.1565 & -1.2467 & 0.1169 & 1.2117 & 0.1916 & 0.9200 & 0.6043 & 1.4235 & 0.0326 & 1.0006 & 0.4442 & 1.3996 & 0.0344 \\
\hline Casella_up & 1.0089 & 0.4748 & 0.6970 & 0.8885 & 0.9737 & 0.4790 & 0.9537 & 0.5209 & 1.3949 & 0.0593 & 0.8874 & 0.6339 & -1.0861 & 0.3168 & 0.6865 & 0.9627 & 0.6751 & 0.9983 \\
\hline Purcell & 0.9329 & 0.5944 & 0.9585 & 0.5093 & 1.5120 & 0.0178 & 1.1731 & 0.2304 & 1.8117 & 0.0000 & 1.5224 & 0.0419 & 1.3894 & 0.0778 & -0.7300 & 0.8874 & -1.0696 & 0.3278 \\
\hline Hernandez & 0.7849 & 0.7771 & 0.7846 & 0.7802 & 0.7146 & 0.9461 & 0.8662 & 0.6710 & -0.8100 & 0.7650 & -0.5778 & 0.9789 & 1.2513 & 0.1718 & 1.4796 & 0.0266 & 1.4019 & 0.0501 \\
\hline Fridman_up & 1.4249 & 0.0169 & 1.5407 & 0.0174 & 1.4397 & 0.0206 & 0.9639 & 0.5449 & 1.7482 & 0.0000 & 1.6100 & 0.0163 & 1.0762 & 0.3145 & 1.3413 & 0.0347 & 1.4113 & 0.0220 \\
\hline Sencan & -0.8460 & 0.9362 & 0.8038 & 0.8235 & 0.8674 & 0.8144 & 1.0328 & 0.4006 & 1.5302 & 0.0011 & 0.7247 & 0.8667 & 0.8838 & 0.7312 & -1.0674 & 0.3182 & 1.5375 & 0.0000 \\
\hline SenMayo & 1.5122 & 0.0023 & 1.4982 & 0.0031 & 1.4624 & 0.0052 & 1.6098 & 0.0013 & 1.8501 & 0.0000 & 1.5100 & 0.0362 & 1.5042 & 0.0023 & 1.4586 & 0.0054 & 1.3239 & 0.0184 \\
\hline
\end{tabular}

\begin{tabular}{|l|l|}
\hline$p$-value & $<0.25$ \\
\hline & $<0.05$ \\
\hline & $<0.01$ \\
\hline
\end{tabular}


bioRxiv preprint doi: https://doi.org/10.1101/2021.12.10.472095; this version posted December 11, 2021. The copyright holder for this preprint (which was not certified by peer review) is the author/funder. All rights reserved. No reuse allowed without permission.

Table 3. Top 20 significantly upregulated genes in the human and murine SASP clusters.

\begin{tabular}{|c|c|c|}
\hline Gene & avg_log2FC & Adj. p-value \\
\hline \multicolumn{3}{|l|}{ Human } \\
\hline S100A9 & 1.974317678 & 0 \\
\hline CXCL8 & 1.817775253 & 0 \\
\hline CST3 & 1.813835295 & 0 \\
\hline TYROBP & 1.742773952 & 0 \\
\hline LST1 & 1.704456515 & 0 \\
\hline FCN1 & 1.704148119 & 0 \\
\hline FCER1G & 1.698071186 & 0 \\
\hline$L Y Z$ & 1.695879021 & 0 \\
\hline CCL3 & 1.68109761 & 0 \\
\hline S100A8 & 1.639167524 & 0 \\
\hline CTSS & 1.605107533 & 0 \\
\hline AIF1 & 1.537560282 & 0 \\
\hline S100A12 & 1.501013381 & 0 \\
\hline SAT1 & 1.475740324 & 0 \\
\hline G0S2 & 1.471768259 & 0 \\
\hline S100A11 & 1.426583167 & 0 \\
\hline$P S A P$ & 1.412156019 & 0 \\
\hline NEAT1 & 1.402008889 & 0 \\
\hline CSTA & 1.346171061 & 0 \\
\hline SERPINA1 & 1.343012763 & 0 \\
\hline \multicolumn{3}{|l|}{ Murine } \\
\hline $\mathrm{Ccl} 2$ & 1.385385456 & $4.4042 E-274$ \\
\hline Cxcl14 & 1.348765531 & 0 \\
\hline Cxcl12 & 1.348099221 & 0 \\
\hline $\mathrm{Hp}$ & 1.32967138 & $2.6772 E-298$ \\
\hline Trf & 1.32483506 & $6.8972 E-280$ \\
\hline Serping1 & 1.304871038 & 0 \\
\hline Mt1 & 1.294617837 & 0 \\
\hline Tmem176b & 1.238330075 & 0 \\
\hline Mt2 & 1.224158694 & 0 \\
\hline lgfbp4 & 1.210905773 & 0 \\
\hline Grem1 & 1.207056724 & 0 \\
\hline Cd302 & 1.195220747 & 0 \\
\hline Apoe & 1.163062993 & 0 \\
\hline Msmp & 1.16240244 & $3.2104 E-194$ \\
\hline Adipoq & 1.140888365 & $7.4114 E-283$ \\
\hline Cyr61 & 1.136426625 & 0 \\
\hline Gas6 & 1.110474329 & 0 \\
\hline Mmp13 & 1.095488296 & 0 \\
\hline Tmem176a & 1.087765581 & 0 \\
\hline Col3a1 & 1.082720154 & $1.1707 E-254$ \\
\hline
\end{tabular}




\section{References}

1. Tchkonia, T., Zhu, Y., van Deursen, J., Campisi, J. \& Kirkland, J. L. Cellular senescence and the senescent secretory phenotype: therapeutic opportunities. The Journal of clinical investigation 123, 966-972; 10.1172/JCl64098 (2013).

2. LeBrasseur, N. K., Tchkonia, T. \& Kirkland, J. L. Cellular Senescence and the Biology of Aging, Disease, and Frailty. Nestle Nutrition Institute workshop series 83, 11-18; 10.1159/000382054 (2015).

3. Swanson, E. C., Manning, B., Zhang, H. \& Lawrence, J. B. Higher-order unfolding of satellite heterochromatin is a consistent and early event in cell senescence. The Journal of cell biology 203, 929-942; 10.1083/jcb.201306073 (2013).

4. Zhu, Y., Armstrong, J. L., Tchkonia, T. \& Kirkland, J. L. Cellular senescence and the senescent secretory phenotype in age-related chronic diseases. Current opinion in clinical nutrition and metabolic care 17, 324-328; 10.1097/MCO.0000000000000065 (2014).

5. Campisi, J. Senescent cells, tumor suppression, and organismal aging: good citizens, bad neighbors. Cell 120, 513-522; 10.1016/j.cell.2005.02.003 (2005).

6. Campisi, J. \& Di d'Adda Fagagna, F. Cellular senescence: when bad things happen to good cells. Nature reviews. Molecular cell biology 8, 729-740; 10.1038/nrm2233 (2007).

7. Farr, J. N. et al. Identification of Senescent Cells in the Bone Microenvironment. Journal of bone and mineral research : the official journal of the American Society for Bone and Mineral Research 31, 1920-1929; 10.1002/jbmr.2892 (2016).

8. Jurk, D. et al. Postmitotic neurons develop a p21-dependent senescence-like phenotype driven by a DNA damage response. Aging cell 11, 996-1004; 10.1111/j.14749726.2012.00870.x (2012).

9. Jurk, D. et al. Chronic inflammation induces telomere dysfunction and accelerates ageing in mice. Nature communications 2, 4172; 10.1038/ncomms5172 (2014).

10. Minamino, T. et al. A crucial role for adipose tissue p53 in the regulation of insulin resistance. Nature medicine 15, 1082-1087; 10.1038/nm.2014 (2009).

11. Wang, E. Senescent human fibroblasts resist programmed cell death, and failure to suppress bcl2 is involved. Cancer research 55, 2284-2292 (1995).

12. Xu, M. et al. Targeting senescent cells enhances adipogenesis and metabolic function in old age. eLife 4, e12997; 10.7554/eLife.12997 (2015).

13. Nelson, G. et al. A senescent cell bystander effect: senescence-induced senescence. Aging cell 11, 345-349; 10.1111/j.1474-9726.2012.00795.x (2012).

14. Acosta, J. C. et al. A complex secretory program orchestrated by the inflammasome controls paracrine senescence. Nature cell biology 15, 978-990; 10.1038/ncb2784 (2013).

15. Coppé, J.-P. et al. Senescence-associated secretory phenotypes reveal cellnonautonomous functions of oncogenic RAS and the p53 tumor suppressor. PLoS biology 6, 2853-2868; 10.1371/journal.pbio.0060301 (2008).

16. Campisi, J. Aging, cellular senescence, and cancer. Annual review of physiology 75, 685705; 10.1146/annurev-physiol-030212-183653 (2013). 
17. Diniz, B. S. et al. Mild cognitive impairment and major depressive disorder are associated with molecular senescence abnormalities in older adults. Alzheimer's \& dementia (New York, N. Y.) 7, e12129; 10.1002/trc2.12129 (2021).

18. Ogrodnik, M. et al. Whole-body senescent cell clearance alleviates age-related brain inflammation and cognitive impairment in mice. Aging cell 20, e13296; 10.1111/acel.13296 (2021).

19. Stout, M. B., Justice, J. N., Nicklas, B. J. \& Kirkland, J. L. Physiological Aging: Links Among Adipose Tissue Dysfunction, Diabetes, and Frailty. Physiology (Bethesda, Md.) 32, 9-19; 10.1152/physiol.00012.2016 (2017).

20. Sławińska, N. \& Krupa, R. Molecular Aspects of Senescence and Organismal Ageing-DNA Damage Response, Telomeres, Inflammation and Chromatin. International journal of molecular sciences 22; 10.3390/ijms22020590 (2021).

21. Boniewska-Bernacka, E., Pańczyszyn, A. \& Klinger, M. Telomeres and telomerase in risk assessment of cardiovascular diseases. Experimental cell research 397, 112361; 10.1016/j.yexcr.2020.112361 (2020).

22. Tchkonia, T., Palmer, A. K. \& Kirkland, J. L. New Horizons: Novel Approaches to Enhance Healthspan Through Targeting Cellular Senescence and Related Aging Mechanisms. The Journal of clinical endocrinology and metabolism 106, e1481-e1487; 10.1210/clinem/dgaa728 (2021).

23. Kirkland, J. L. \& Tchkonia, T. Senolytic drugs: from discovery to translation. Journal of internal medicine 288, 518-536; 10.1111/joim.13141 (2020).

24. Hickson, L. J. et al. Senolytics decrease senescent cells in humans: Preliminary report from a clinical trial of Dasatinib plus Quercetin in individuals with diabetic kidney disease. EBioMedicine 47, 446-456; 10.1016/j.ebiom.2019.08.069 (2019).

25. Lähnemann, D. et al. Eleven grand challenges in single-cell data science. Genome biology 21, 31; 10.1186/s13059-020-1926-6 (2020).

26. Borodkina, A. V., Deryabin, P. I., Giukova, A. A. \& Nikolsky, N. N. "Social Life" of Senescent Cells: What Is SASP and Why Study It? Acta naturae 10, 4-14 (2018).

27. Farr, J. N. et al. Effects of Age and Estrogen on Skeletal Gene Expression in Humans as Assessed by RNA Sequencing. PloS one 10, e0138347; 10.1371/journal.pone.0138347 (2015).

28. Weivoda, M. M. et al. Identification of osteoclast-osteoblast coupling factors in humans reveals links between bone and energy metabolism. Nature communications 11, 87; 10.1038/s41467-019-14003-6 (2020).

29. Han, H. et al. TRRUST: a reference database of human transcriptional regulatory interactions. Scientific reports 5, 11432; 10.1038/srep11432 (2015).

30. Shannon, P. et al. Cytoscape: a software environment for integrated models of biomolecular interaction networks. Genome research 13, 2498-2504; 10.1101/gr.1239303 (2003).

31. Subramanian, A. et al. Gene set enrichment analysis: a knowledge-based approach for interpreting genome-wide expression profiles. Proceedings of the National Academy of Sciences of the United States of America 102, 15545-15550; 10.1073/pnas.0506580102 (2005).

32. Shi, L. et al. Genome-wide transcriptomic analysis of microglia reveals impaired responses in aged mice after cerebral ischemia. Journal of cerebral blood flow and metabolism : official 
journal of the International Society of Cerebral Blood Flow and Metabolism 40, S49-S66; 10.1177/0271678X20925655 (2020).

33. Guo, X. et al. Age attenuates the transcriptional changes that occur with sleep in the medial prefrontal cortex. Aging cell 18, e13021; 10.1111/acel.13021 (2019).

34. Kwapis, J. L. et al. Epigenetic regulation of the circadian gene Per1 contributes to agerelated changes in hippocampal memory. Nature communications 9, 3323; 10.1038/s41467018-05868-0 (2018).

35. Tabula Muris Consortium. A single-cell transcriptomic atlas characterizes ageing tissues in the mouse. Nature 583, 590-595; 10.1038/s41586-020-2496-1 (2020).

36. Schaum, N. et al. Ageing hallmarks exhibit organ-specific temporal signatures. Nature 583, 596-602; 10.1038/s41586-020-2499-y (2020).

37. Farr, J. N. et al. Targeting cellular senescence prevents age-related bone loss in mice. Nature medicine 23, 1072-1079; 10.1038/nm.4385 (2017).

38. Hickson, L. J. et al. Corrigendum to 'Senolytics decrease senescent cells in humans: Preliminary report from a clinical trial of Dasatinib plus Quercetin in individuals with diabetic kidney disease' EBioMedicine 47 (2019) 446-456. EBioMedicine 52, 102595; 10.1016/j.ebiom.2019.12.004 (2020).

39. Zhu, Y. et al. The Achilles' heel of senescent cells: from transcriptome to senolytic drugs. Aging cell 14, 644-658; 10.1111/acel.12344 (2015).

40. Casella, G. et al. Transcriptome signature of cellular senescence. Nucleic acids research 47, 11476; 10.1093/nar/gkz879 (2019).

41. Purcell, M., Kruger, A. \& Tainsky, M. A. Gene expression profiling of replicative and induced senescence. Cell cycle (Georgetown, Tex.) 13, 3927-3937; 10.4161/15384101.2014.973327 (2014).

42. Hernandez-Segura, A. et al. Unmasking Transcriptional Heterogeneity in Senescent Cells. Current biology : CB 27, 2652-2660.e4; 10.1016/j.cub.2017.07.033 (2017).

43. Fridman, A. L. \& Tainsky, M. A. Critical pathways in cellular senescence and immortalization revealed by gene expression profiling. Oncogene 27, 5975-5987; 10.1038/onc.2008.213 (2008).

44. Jochems, F. et al. The Cancer SENESCopedia: A delineation of cancer cell senescence. Cell reports 36, 109441; 10.1016/j.celrep.2021.109441 (2021).

45. Johmura, Y. et al. Senolysis by glutaminolysis inhibition ameliorates various age-associated disorders. Science (New York, N.Y.) 371, 265-270; 10.1126/science.abb5916 (2021).

46. Oetjen, K. A. et al. Human bone marrow assessment by single-cell RNA sequencing, mass cytometry, and flow cytometry. JCl insight 3; 10.1172/jci.insight.124928 (2018).

47. Magalhães, J. P. de, Curado, J. \& Church, G. M. Meta-analysis of age-related gene expression profiles identifies common signatures of aging. Bioinformatics (Oxford, England) 25, 875-881; 10.1093/bioinformatics/btp073 (2009).

48. Avelar, R. A. et al. A multidimensional systems biology analysis of cellular senescence in aging and disease. Genome biology 21, 91; 10.1186/s13059-020-01990-9 (2020).

49. Razdan, N., Vasilopoulos, T. \& Herbig, U. Telomere dysfunction promotes transdifferentiation of human fibroblasts into myofibroblasts. Aging cell 17, e12838; 10.1111/acel.12838 (2018). 
50. Jin, S. et al. Inference and analysis of cell-cell communication using CellChat. Nature communications 12, 1088; 10.1038/s41467-021-21246-9 (2021).

51. Landherr, A., Friedl, B. \& Heidemann, J. A Critical Review of Centrality Measures in Social Networks. Bus Inf Syst Eng 2, 371-385; 10.1007/s12599-010-0127-3 (2010).

52. Baryawno, N. et al. A Cellular Taxonomy of the Bone Marrow Stroma in Homeostasis and Leukemia. Cell 177, 1915-1932.e16; 10.1016/j.cell.2019.04.040 (2019).

53. Kale, A., Sharma, A., Stolzing, A., Desprez, P.-Y. \& Campisi, J. Role of immune cells in the removal of deleterious senescent cells. Immunity \& Ageing : I \& A 17; 10.1186/s12979-02000187-9 (2020).

54. Schafer, M. J. et al. The senescence-associated secretome as an indicator of age and medical risk. JCl insight 5; 10.1172/jci.insight.133668 (2020).

55. Lopes-Paciencia, S. et al. The senescence-associated secretory phenotype and its regulation. Cytokine 117, 15-22; 10.1016/j.cyto.2019.01.013 (2019).

56. Leon, K. E. et al. DOT1L modulates the senescence-associated secretory phenotype through epigenetic regulation of IL1A. The Journal of cell biology 220; 10.1083/jcb.202008101 (2021).

57. Buj, R., Leon, K. E., Anguelov, M. A. \& Aird, K. M. Suppression of p16 alleviates the senescence-associated secretory phenotype. Aging 13, 3290-3312; 10.18632/aging.202640 (2021).

58. Andriani, G. A. et al. Whole Chromosome Instability induces senescence and promotes SASP. Scientific reports 6, 35218; 10.1038/srep35218 (2016).

59. Kumari, R. \& Jat, P. Mechanisms of Cellular Senescence: Cell Cycle Arrest and Senescence Associated Secretory Phenotype. Frontiers in cell and developmental biology 9, 645593; 10.3389/fcell.2021.645593 (2021).

60. Ma, S. et al. Single-cell transcriptomic atlas of primate cardiopulmonary aging. Cell research 31, 415-432; 10.1038/s41422-020-00412-6 (2021).

61. Cuollo, L., Antonangeli, F., Santoni, A. \& Soriani, A. The Senescence-Associated Secretory Phenotype (SASP) in the Challenging Future of Cancer Therapy and Age-Related Diseases. Biology 9; 10.3390/biology9120485 (2020).

62. Khosla, S., Farr, J. N., Tchkonia, T. \& Kirkland, J. L. The role of cellular senescence in ageing and endocrine disease. Nature reviews. Endocrinology 16, 263-275; 10.1038/s41574-020-0335-y (2020).

63. Leon, K. E., Tangudu, N. K., Aird, K. M. \& Buj, R. Loss of p16: A Bouncer of the Immunological Surveillance? Life (Basel, Switzerland) 11; 10.3390/life11040309 (2021).

64. Kolesnichenko, M. et al. Transcriptional repression of NFKBIA triggers constitutive IKK- and proteasome-independent p65/RelA activation in senescence. The EMBO journal 40, e104296; 10.15252/embj.2019104296 (2021).

65. Docherty, M.-H., O'Sullivan, E. D., Bonventre, J. V. \& Ferenbach, D. A. Cellular Senescence in the Kidney. Journal of the American Society of Nephrology : JASN 30, 726-736; 10.1681/ASN.2018121251 (2019).

66. Amaya-Montoya, M., Pérez-Londoño, A., Guatibonza-García, V., Vargas-Villanueva, A. \& Mendivil, C. O. Cellular Senescence as a Therapeutic Target for Age-Related Diseases: A Review. Advances in therapy 37, 1407-1424; 10.1007/s12325-020-01287-0 (2020). 
67. Kimmel, J. C., Yi, N., Roy, M., Hendrickson, D. G. \& Kelley, D. R. Differentiation reveals latent features of aging and an energy barrier in murine myogenesis. Cell reports 35, 109046; 10.1016/j.celrep.2021.109046 (2021).

68. lacono, G. et al. bigSCale: an analytical framework for big-scale single-cell data. Genome research 28, 878-890; 10.1101/gr.230771.117 (2018).

69. Blencowe, M. et al. Network modeling of single-cell omics data: challenges, opportunities, and progresses. Emerging topics in life sciences 3, 379-398; 10.1042/etls20180176 (2019).

70. Iacono, G., Massoni-Badosa, R. \& Heyn, H. Single-cell transcriptomics unveils gene regulatory network plasticity. Genome biology 20, 110; 10.1186/s13059-019-1713-4 (2019).

71. Salminen, A. \& Kaarniranta, K. Control of p53 and NF-KB signaling by WIP1 and MIF: role in cellular senescence and organismal aging. Cellular signalling 23, 747-752; 10.1016/j.cellsig.2010.10.012 (2011).

72. Schmid, N. et al. Insights into replicative senescence of human testicular peritubular cells. Scientific reports 9, 15052; 10.1038/s41598-019-51380-w (2019).

73. Zhang, Y. et al. Macrophage migration inhibitory factor activates the inflammatory response in joint capsule fibroblasts following post-traumatic joint contracture. Aging 13, 5804-5823; 10.18632/aging.202505 (2021).

74. Yamada, C. et al. Glycyrrhizin mitigates inflammatory bone loss and promotes expression of senescence-protective sirtuins in an aging mouse model of periprosthetic osteolysis.

Biomedicine \& pharmacotherapy = Biomedecine \& pharmacotherapie 138, 111503; 10.1016/j.biopha.2021.111503 (2021).

75. Florez-Sampedro, L., Soto-Gamez, A., Poelarends, G. J. \& Melgert, B. N. The role of MIF in chronic lung diseases: looking beyond inflammation. American journal of physiology. Lung cellular and molecular physiology 318, L1183-L1197; 10.1152/ajplung.00521.2019 (2020).

76. Zhang, Y. et al. Macrophage migration inhibitory factor rejuvenates aged human mesenchymal stem cells and improves myocardial repair. Aging 11, 12641-12660; 10.18632/aging.102592 (2019).

77. Hu, Y., Xia, W. \& Hou, M. Macrophage migration inhibitory factor serves a pivotal role in the regulation of radiation-induced cardiac senescencethrough rebalancing the microRNA34a/sirtuin 1 signaling pathway. International journal of molecular medicine 42, 2849-2858; 10.3892/ijmm.2018.3838 (2018).

78. Noe, J. T. \& Mitchell, R. A. MIF-Dependent Control of Tumor Immunity. Frontiers in immunology 11, 609948; 10.3389/fimmu.2020.609948 (2020).

79. Ghosh, S., Jiang, N., Farr, L., Ngobeni, R. \& Moonah, S. Parasite-Produced MIF Cytokine: Role in Immune Evasion, Invasion, and Pathogenesis. Frontiers in immunology 10, 1995; 10.3389/fimmu.2019.01995 (2019).

80. Coppé, J.-P., Desprez, P.-Y., Krtolica, A. \& Campisi, J. The senescence-associated secretory phenotype: the dark side of tumor suppression. Annual review of pathology 5, 99118; 10.1146/annurev-pathol-121808-102144 (2010).

81. Kirkland, J. L. \& Tchkonia, T. Cellular Senescence: A Translational Perspective. EBioMedicine 21, 21-28; 10.1016/j.ebiom.2017.04.013 (2017).

82. Birch, J. \& Gil, J. Senescence and the SASP: many therapeutic avenues. Genes \& development 34, 1565-1576; 10.1101/gad.343129.120 (2020). 
83. Salotti, J. \& Johnson, P. F. Regulation of senescence and the SASP by the transcription factor C/EBPß. Experimental gerontology 128, 110752; 10.1016/j.exger.2019.110752 (2019).

84. Basisty, N. et al. A proteomic atlas of senescence-associated secretomes for aging biomarker development. PLoS biology 18, e3000599; 10.1371/journal.pbio.3000599 (2020).

85. Yousefzadeh, M. J. et al. Mouse Models of Accelerated Cellular Senescence. Methods in molecular biology (Clifton, N.J.) 1896, 203-230; 10.1007/978-1-4939-8931-7_17 (2019).

86. Dodig, S., Čepelak, I. \& Pavić, I. Hallmarks of senescence and aging. Biochemia medica 29, 30501; 10.11613/BM.2019.030501 (2019).

87. Kiss, T. et al. Single-cell RNA sequencing identifies senescent cerebromicrovascular endothelial cells in the aged mouse brain. GeroScience 42, 429-444; 10.1007/s11357-02000177-1 (2020).

88. Lasry, A. \& Ben-Neriah, Y. Senescence-associated inflammatory responses: aging and cancer perspectives. Trends in immunology 36, 217-228; 10.1016/j.it.2015.02.009 (2015).

89. Zhang, W. et al. Lycorine hydrochloride suppresses stress-induced premature cellular senescence by stabilizing the genome of human cells. Aging cell 20, e13307; 10.1111/acel.13307 (2021).

90. Mootha, V. K. et al. PGC-1alpha-responsive genes involved in oxidative phosphorylation are coordinately downregulated in human diabetes. Nature genetics 34, 267-273; 10.1038/ng1180 (2003).

91. Eckhardt, B. A. et al. Accelerated osteocyte senescence and skeletal fragility in mice with type 2 diabetes. JCl insight 5; 10.1172/jci.insight.135236 (2020).

92. Kosinsky, R. L. et al. RNF20 and RNF40 regulate vitamin D receptor-dependent signaling in inflammatory bowel disease. Cell death and differentiation; 10.1038/s41418-021-00808-w (2021).

93. Alquicira-Hernandez, J. \& Powell, J. E. Nebulosa recovers single cell gene expression signals by kernel density estimation. Bioinformatics (Oxford, England); 10.1093/bioinformatics/btab003 (2021).

94. Qiu, X. et al. Reversed graph embedding resolves complex single-cell trajectories. Nature methods 14, 979-982; 10.1038/nmeth.4402 (2017).

95. Bunis, D. G., Andrews, J., Fragiadakis, G. K., Burt, T. D. \& Sirota, M. dittoSeq: Universal User-Friendly Single-Cell and Bulk RNA Sequencing Visualization Toolkit. Bioinformatics (Oxford, England); 10.1093/bioinformatics/btaa1011 (2020).

96. Kolberg, L., Raudvere, U., Kuzmin, I., Vilo, J. \& Peterson, H. gprofiler2 -- an R package for gene list functional enrichment analysis and namespace conversion toolset g:Profiler. F1000Research 9; 10.12688/f1000research.24956.2 (2020).

97. Trapnell, C. et al. The dynamics and regulators of cell fate decisions are revealed by pseudotemporal ordering of single cells. Nature biotechnology 32, 381-386; 10.1038/nbt.2859 (2014).

98. Reid, J. E. \& Wernisch, L. Pseudotime estimation: deconfounding single cell time series. Bioinformatics (Oxford, England) 32, 2973-2980; 10.1093/bioinformatics/btw372 (2016). 
bioRxiv preprint doi: https://doi.org/10.1101/2021.12.10.472095; this version posted December 11, 2021. The copyright holder for this preprint (which was not certified by peer review) is the author/funder. All rights reserved. No reuse allowed without permission.

99. Reimand, J. et al. Pathway enrichment analysis and visualization of omics data using $\mathrm{g}:$ Profiler, GSEA, Cytoscape and EnrichmentMap. Nature protocols 14, 482-517; 10.1038/s41596-018-0103-9 (2019). 